\title{
Thermodynamics and dynamics of a 1-D gravitational system
}

\author{
P. Valageas
}

Service de Physique Théorique, CEN Saclay, 91191 Gif-sur-Yvette, France

e-mail: valageas@spht.saclay.cea.fr

Received 4 November 2005 / Accepted 11 January 2006

\section{ABSTRACT}

Aims. The dynamics of large-scale structure formation in the universe by gravitational instability still presents many open issues and is mostly studied through numerical simulations. This motivates the study of simpler models which can be investigated by analytical means in order to understand the main processes at work. Thus, we describe here a one-dimensional self-gravitating system derived from the cosmological context, which leads to an effective external potential in addition to the standard gravitational self-interaction. As a first step we consider small times so that the expansion can be neglected. Then we present a thermodynamical analysis of this system as well as the stability properties of the associated hydrodynamical and collisionless systems.

Methods. We consider the mean field limit (i.e. continuum limit) to perform an analytical study.

Results. We find a second-order phase transition at $T_{c 1}$ from an homogeneous equilibrium at high temperature to a clustered phase (with a density peak at one of the boundaries of the system) at low temperature. There also exists an infinite series of unstable equilibria which appear at lower temperatures $T_{c n}$, reflecting the scale-free nature of the gravitational interaction and the usual Jeans instability. We find that, as for the similar HMF (Hamiltonian mean field) model, all three micro-canonical, canonical and grand-canonical ensembles agree with each other, as well as with the stability properties associated with a hydrodynamical approach. On the other hand, the collisionless dynamics governed by the Vlasov equation yields the same results except that at low $T$ the equilibrium associated with two density peaks (one at each boundary) becomes stable.

Key words. gravitation - cosmology: theory - large-scale structure of Universe

\section{Introduction}

In standard cosmological scenarios the large scale structures we observe in the present universe (such as clusters, filaments or voids) have formed through the amplification by gravitational instability of small primordial perturbations, see Peebles (1980). Moreover, the amplitude of these density fluctuations increases at small scales as in the CDM model (Peebles 1982) which leads to a hierarchical process where smaller scales become non-linear first. Then, at large scales or at early times one can use a perturbative approach to study the evolution of initial fluctuations (Fry 1984; Goroff et al. 1986; Bernardeau 1992; Valageas 2001). Next, the weakly non-linear regime may be investigated through the Zeldovich approximation (Shandarin \& Zeldovich 1989) or the adhesion model (Gurbatov et al. 1989). However, the highly non-linear regime (which corresponds to collapsed structures such as clusters of galaxies) has mostly remained out of reach of systematic approaches. Thus, one may use the Press-Schechter approximation (Press \& Schechter 1974) or the excursion-set formalism of Bond et al. (1991) to obtain the statistics of just-virialized halos or the saddle-point approach of Valageas (2002) for rare voids. These approximations focus on specific objects which may be "recognized" from individual features in the linear density field itself and do not follow the system as a whole through its non-linear evolution. A systematic method to do so was recently developed in Valageas (2004) but its application to the collisionless dynamics has not been performed yet for the highly non-linear regime. Therefore, the non-linear regime of cosmological structure formation is mostly studied through numerical simulations.

In order to simplify this difficult problem one can investigate one-dimensional (1-D) systems which are easier to study both through numerical and analytical approaches. In fact, 1-D systems such as the system of parallel mass sheets (Camm 1950) have been studied for a long time to explore the evolution of isolated $\mathrm{N}$-body systems which only interact through classical gravity. In particular, they have been used to investigate relaxation processes and to test Lynden-Bell's prediction for the final state of violent relaxation (Lynden-Bell 1967). Such 1-D systems can already exhibit complex behaviours. For instance, Luwel \& Severne (1985) found that collisional effects are not sufficient to relax the stationary waterbag configuration towards thermodynamical equilibrium whereas Rouet \& Feix (1999) showed that holes in phase-space in the initial distribution function can persist over long times and prevent efficient relaxation. On the other hand, the system can break up into 
smaller clusters as in Hohl \& Feix (1967) or develop a fractal structure as in Koyama \& Konishi (2001).

In this paper, we consider the 1-D gravitational system obtained by studying 1-D density fluctuations in a 3-D cosmological background. This model has already been studied mostly through numerical simulation in Aurell \& Fanelli (2001), Aurell et al. (2001) and Fanelli \& Aurell (2002), both with and without cosmological expansion. Here we restrict ourselves to time-scales much smaller than the Hubble time so that the expansion of the universe can be neglected and we focus on a mean field analysis (i.e. a continuum limit) which is relevant in the cosmological context. We study the thermodynamics and stability of this system, similarly to the theoretical analysis of Chavanis et al. (2005) performed for the HMF model (defined by a cosine interaction) which shows a similar behaviour. We introduce this model in Sect. 2, where the effect of the cosmological background is seen to reduce to an effective external potential $V$ which balances the gravitational self-interaction $\Phi$ so that the homogeneous state is an equilibrium solution. Next, we perform a thermodynamical analysis of this system in Sect. 3, for the micro-canonical, canonical and grand-canonical ensembles. We study both the possible equilibrium states and their stability properties. Then, in Sect. 4 we investigate the stability of such equilibria within an hydrodynamical framework whereas we consider the collisionless case as described by the Vlasov dynamics in Sect. 5. Finally, we present our conclusions in Sect. 6.

\section{A 1-D cosmological gravitational system}

The formation of large-scale structures in the universe through gravitational instability is described by the Vlasov-Poisson system which applies to a collisionless fluid in the continuum limit where the mass $m$ of the particles goes to zero. Thus, the distribution function (phase-space density) $f(\boldsymbol{x}, \boldsymbol{v}, t)$, where we note $\boldsymbol{x}$ the comoving coordinate of a particle and $\boldsymbol{v}$ its peculiar velocity defined by $\boldsymbol{v}=a^{2} \dot{\boldsymbol{x}}$ (and $a(t)$ is the cosmological scale-factor), obeys the Vlasov equation (see Peebles 1980):

$\frac{\partial f}{\partial t}+\frac{\boldsymbol{v}}{a^{2}} \cdot \frac{\partial f}{\partial \boldsymbol{x}}-\frac{\partial \phi}{\partial \boldsymbol{x}} \cdot \frac{\partial f}{\partial \boldsymbol{v}}=0$

while the gravitational potential $\phi$ satisfies the Poisson equation:

$\Delta \phi=\frac{4 \pi \mathcal{G}}{a}(\rho-\bar{\rho})$ with $\rho(\boldsymbol{x}, t)=\int f(\boldsymbol{x}, \boldsymbol{v}, t) \mathrm{d} \boldsymbol{v}$.

Here we introduced the comoving density $\rho(\boldsymbol{x}, t)$ and its average $\bar{\rho}$ (which is independent of time), and we note $\mathcal{G}$ Newton's constant. The fact that the Poisson equation involves the density perturbation $(\rho-\bar{\rho})$ rather than the total density $\rho$ comes from the change from physical coordinates to comoving coordinates. In particular, for an homogeneous universe which merely follows the Hubble flow we have $\phi=0$ and $f(\boldsymbol{x}, \boldsymbol{v}, t)=$ $\bar{\rho} \delta_{D}(\boldsymbol{v})$ where $\delta_{D}$ is Dirac's distribution. Alternatively, the constant term $-\bar{\rho}$ may be interpreted as a cosmological constant, see de Vega \& Siebert (2005a,b) for a study of the statistical mechanics in the 3-D case. If we consider planar perturbations along the axis $x: f(\boldsymbol{x}, \boldsymbol{v}, t)=f(x, v, t) \delta_{D}\left(\boldsymbol{v}_{\perp}\right)$, we obtain a onedimensional (1-D) problem:

$\frac{\partial f}{\partial t}+\frac{v}{a^{2}} \cdot \frac{\partial f}{\partial x}-\frac{\partial \phi}{\partial x} \cdot \frac{\partial f}{\partial v}=0, \quad \frac{\partial^{2} \phi}{\partial x^{2}}=\frac{4 \pi \mathcal{G}}{a}(\rho-\bar{\rho})$.

Let us moreover restrict ourselves to periodic systems of period $2 L$ which are symmetric with respect to the origin $x=0$. Then, by symmetry the force $F=-\partial \phi / \partial x$ vanishes at the origin, $F(x=0)=0$, and the gravitational potential can be integrated as:

$F(x)=-\frac{\partial \phi}{\partial x}=\frac{2 \pi \mathcal{G}}{a} \int_{0}^{L} \mathrm{~d} x^{\prime}(\rho-\bar{\rho})\left(x^{\prime}\right) \operatorname{sign}\left(x^{\prime}-x\right)$,

and:

$\phi(x)=\frac{2 \pi \mathcal{G}}{a} \int_{0}^{L} \mathrm{~d} x^{\prime}(\rho-\bar{\rho})\left(x^{\prime}\right)\left|x-x^{\prime}\right|$,

where we restrict ourselves to $0 \leq x \leq L$. If we consider timescales which are much smaller than the Hubble time $H^{-1}=a / \dot{a}$ we can neglect the time dependence of the scale-factor $a(t)$ and taking $a=1$ and $g=2 \pi \mathcal{G}$ we can write the full potential $\phi$ as:

$\phi=\Phi+V$ with $\left\{\begin{array}{l}\Phi=g \int_{0}^{L} \mathrm{~d} x^{\prime} \rho\left(x^{\prime}\right)\left|x-x^{\prime}\right| \\ V(x)=-g \bar{\rho}\left[\left(x-\frac{L}{2}\right)^{2}+\frac{L^{2}}{4}\right]\end{array}\right.$

where we split $\phi$ into a standard gravitational "interaction" term $\Phi$ and an "external" potential $V$. Thus, the associated discrete system of $N$ particles of finite mass $m$ is described by the Hamiltonian $\mathcal{H}_{\mathcal{N}}$ :

$$
\begin{aligned}
\mathcal{H}_{\mathcal{N}}= & \sum_{i=1}^{N} \frac{1}{2} m v_{i}^{2}+g m^{2} \sum_{i>j}\left|x_{i}-x_{j}\right| \\
& -g \bar{\rho} m \sum_{i=1}^{N}\left[\left(x_{i}-\frac{L}{2}\right)^{2}+\frac{L^{2}}{4}\right] .
\end{aligned}
$$

Alternatively, the mean field approximation to the $N$-body system (7) is given by the potential (6) and the Vlasov-Poisson equations. The important feature of this one-dimensional static cosmology (OSC) model which is due to its cosmological setting is the appearance of the "background" potential $V(x)$ which ensures that the homogeneous configuration $\rho=\bar{\rho}$ is a solution of the equation of motion. This is the counterpart of the Hubble flow. The OSC model defined by Eqs. (7) or (6) was studied numerically in Aurell et al. (2001) and Aurell \& Fanelli (2001) (where it is called a "frictionless model") for random (Brownian motion) and non-random (sinusoidal) initial conditions. In this article, we shall consider the thermodynamics of the continuous system (6), which can also be seen as the mean field approximation to Eq. (7). This is a system of finite size $L$ with $0 \leq x \leq L$. However, it can be useful to remember that it can also be understood as a periodic system of size $2 L$ which is symmetric with respect to the origin $x=0$.

Here, we should add a few words about the limitations of this OSC model with regard to cosmology. The real universe is obviously three-dimensional and some important features such as the support of self-gravitating halos by rotation (as for spiral galaxies) cannot be studied through 1-D models. Thus, although the early stages of non-linear evolution are governed by 
1-D collapse (formation of pancakes, Shandarin \& Zeldovich 1989) the highly non-linear regime which is of interest to us is truly three-dimensional. However, we can expect that key processes associated with the scale-free nature of the gravitational interaction are common to 1-D, 2-D and 3-D systems. For instance Munshi et al. (1999) find that 2-D and 3-D cosmological simulations exhibit similar behaviours in the highly nonlinear regime. In particular, 1-D systems should help to check whether the density profiles of virialized halos show a universal shape, for a wide class of initial conditions, and whether the power-spectrum of density fluctuations saturates into the non-linear regime or exhibits a power-law growth which could depend on initial conditions. A study of 1-D systems such as the OSC model is particularly timely as modern supercomputers are beginning to allow 1-D cosmological simulations in phase-space instead of the usual $N$-body techniques (Alard \& Colombi 2005).

On the other hand, the use of a finite size domain $0 \leq x \leq L$ (which is equivalent to periodic boundary conditions of period $2 L$ with symmetry with respect to $x=0$ ) is customary to studies of gravitational systems (e.g. Padmanabhan 1990). Within the cosmological context, characterized by a hierarchical scenario where smaller scales turn non-linear first, the size $L$ has actually a physical meaning. It should be interpreted as the wavelength which turns non-linear at the time of interest. Indeed, larger scales which are still linear follow the Hubble flow (up to small perturbations) while smaller scales have relaxed within virialized halos. Thus, to this scale is also associated a characteristic time $t_{\mathrm{H}}$ given by the age of the universe when $L$ enters the non-linear regime. Today this yields $t_{\mathrm{H}} \sim 10^{10} \mathrm{yrs}$ and $L \sim 5 \mathrm{Mpc}$. Then, the expansion of the universe can be neglected if we restrict ourselves to length and time scales much smaller than $L$ and $t_{\mathrm{H}}$. Of course, this may also prevent the system to reach thermodynamical equilibrium which can require very long timescales (increasing algebraically with the number $N$ of particles, Chavanis 2006).

However, this article should only be seen as a first step in the study of the OSC system. We think that this model is also interesting in its own right as it should exhibit the features associated with a long-range scale-free interaction, which is an active domain of research. Besides, the background $-\bar{\rho}$ in Poisson Eq. (2) which arises from the cosmological context also leads to interesting transition phenomena as described in Sect. 3 below.

\section{Thermodynamical analysis}

\subsection{Statistical equilibrium}

\subsubsection{Micro-canonical ensemble}

\section{Equilibrium profiles:}

In the micro-canonical ensemble the statistical equilibrium is obtained by maximizing the Boltzmann entropy $S[f]$ at fixed energy $E$ and mass $M$, given by (Padmanabhan 1990; Chavanis et al. 2005):

$$
S=-\int \mathrm{d} x \mathrm{~d} v f \ln f, \quad E=\int \mathrm{d} x \mathrm{~d} v f\left(\frac{v^{2}}{2}+\frac{\Phi}{2}+V\right)
$$

and:

$$
M=\bar{\rho} L=\int \mathrm{d} x \mathrm{~d} v f
$$

Using Lagrange multipliers this yields the usual MaxwellBoltzmann distribution:

$f(x, v) \propto \mathrm{e}^{-\beta\left[v^{2} / 2+\phi(x)\right]}$ and $\rho(x) \propto \mathrm{e}^{-\beta \phi(x)}$,

where we introduced the inverse temperature $\beta=1 / T$. Let us define the offset $\bar{\varphi}$ such that:

$\phi=\varphi+\bar{\varphi}$ with $\rho=\bar{\rho} \mathrm{e}^{-\beta \varphi}$ and $\frac{\mathrm{d}^{2} \varphi}{\mathrm{d} x^{2}}=2 g(\rho-\bar{\rho})$.

Therefore, the thermodynamical equilibrium is set by the equations:

$\frac{\mathrm{d}^{2} \varphi}{\mathrm{d} x^{2}}=2 g \bar{\rho}\left(\mathrm{e}^{-\beta \varphi}-1\right) \quad$ and $\quad \varphi^{\prime}(0)=\varphi^{\prime}(L)=0$,

where the boundary conditions are obtained from Eq. (4). Without the constant term -1 in its r.h.s. Eq. (12) reduces to the usual Lane-Emden equation which arises for isolated selfgravitating isothermal gaseous spheres (e.g., Chandrasekhar 1942; Binney \& Tremaine 1987). It is convenient to introduce the Jeans length $L_{\mathrm{J}}$ (Binney \& Tremaine 1987) and the ratio $\zeta_{L}$ :

$L_{\mathrm{J}}=(2 g \bar{\rho} \beta)^{-1 / 2}, \quad \zeta_{L}=\frac{L}{L_{\mathrm{J}}}=\sqrt{2 g \bar{\rho} \beta} L$

in order to define the dimensionless quantities:

$\zeta=\frac{x}{L} \zeta_{L}, \quad \psi=\beta \varphi$ and $\eta=\frac{\rho}{\bar{\rho}}$, whence $\eta=\mathrm{e}^{-\psi}$.

Then, the modified Lane-Emden Eq. (12) reads:

$\frac{\mathrm{d}^{2} \psi}{\mathrm{d} \zeta^{2}}=\mathrm{e}^{-\psi}-1$ and $\psi^{\prime}(0)=\psi^{\prime}\left(\zeta_{L}\right)=0$.

This is the equation of motion of a particle of mass $m=1$ and coordinate $\psi$ as a function of time $\zeta$ within a potential $\mathcal{V}(\psi)=\mathrm{e}^{-\psi}+\psi-1$, with zero velocity at initial $(\zeta=0)$ and final $\left(\zeta_{L}\right)$ times. The case where the particle is at rest at the bottom $\psi=0$ of the potential $\mathcal{V}(\psi)$ is always a solution of Eq. (15). This corresponds to the uniform density $\rho=\bar{\rho}$. As the temperature $T$ decreases, while $\beta$ and $\zeta_{L}$ grow, new solutions appear as the particle now has time to perform one or several oscillations in the potential well $\mathcal{V}(\psi)$. This yields a series of critical temperatures $T_{c n}$, or $\beta_{c n}$, associated with the appearance of a solution with $n$ half-oscillations. They can be obtained by expanding the potential $\mathcal{V}(\psi)$ around $\psi=0$ up to quadratic order. This yields:

$\mathcal{V}(\psi) \simeq \frac{\psi^{2}}{2}$, whence $\psi^{\prime \prime}+\psi=0$ and $\psi=\psi_{0} \cos \zeta$

where we used $\psi^{\prime}(0)=0$. Next, the second constraint $\psi^{\prime}\left(\zeta_{L}\right)=$ 0 implies:

$\zeta_{L}=n \pi \quad$ whence $\beta_{c n}=\frac{n^{2} \pi^{2}}{2 g \bar{\rho} L^{2}}, \quad T_{c n}=\frac{2 g \bar{\rho} L^{2}}{n^{2} \pi^{2}}$.

If $n$ is odd this corresponds to $n$ half-oscillations starting at $\psi_{0}$ and ending at $-\psi_{0}$ whereas if $n$ is even it corresponds to $n / 2$ 
full oscillations starting and ending at $\psi_{0}$. As the temperature decreases below $T_{c n}$ the oscillations get wider and take "more time". In the following we shall label by " $n$ " the solution with $n$ half-oscillations which starts at $\psi(0)=\psi_{-}<0$ and by $-n$ the solution with $n$ half-oscillations which starts at $\psi(0)=\psi_{+}>0$. From the last equation in (10) or (14) we see for instance that the mode $n=2$ shows two density peaks at $x=0$ and $x=L$ while the mode $n=-2$ shows only one density peak at $x=L / 2$ (but two underdense minima at $x=0$ and $x=L$ ). We shall call the homogeneous equilibrium solution $\rho=\bar{\rho}$ as the mode $n=0$. On the other hand, since the differential equation in (15) does not depend on $\beta$ or $n$ (which only appear in the boundary conditions) all modes $\pm n$ can be written in terms of the mode $n=1$ at a different temperature $T_{1}$ given by $\zeta_{L 1}=\zeta_{L} / n$ or $\beta_{1}=\beta / n^{2}$. Thus, we have:

$$
\begin{aligned}
\psi_{n}(\zeta ; \beta) & =\psi_{1}\left(\zeta ; \frac{\beta}{n^{2}}\right) \\
\psi_{-n}(\zeta ; \beta) & =\psi_{1}\left(\zeta-\frac{\zeta_{L}}{n} ; \frac{\beta}{n^{2}}\right)
\end{aligned}
$$

and similar relations for the overdensity $\eta$. Here we considered that all solutions are extended over the full real axis to yield solutions which are periodic of period $2 L$ and are symmetric with respect to $x=0$, in agreement with Sect. 2 . This merely amounts to follow the motion of the particle within the potential well $\mathcal{V}(\psi)$ over all times. The periodicity and symmetry of $\psi(\zeta)$ implies that we can expand the solution as:

$\psi(\zeta)=\sum_{k=0}^{\infty} a_{k} \cos \left(k \pi \frac{\zeta}{\zeta_{L}}\right)$

This automatically satisfies the boundary conditions (15). As seen in Eqs. (18), (19) it is sufficient to consider the case $n=1$. Then, in order to study the behaviour close to the critical temperature we can substitute the expansion (20) into the differential Eq. (15) up to the required order over $\left(\beta-\beta_{c 1}\right)$. We obtain up to order $\left(\beta-\beta_{c 1}\right)$ :

$a_{0}=\frac{a_{1}^{2}}{4}, \quad a_{2}=-\frac{a_{1}^{2}}{12}, \quad a_{1}=-\sqrt{12 \frac{\beta-\beta_{c 1}}{\beta_{c 1}}}$,

while higher-order terms $a_{k}$ are of order $a_{k} \sim a_{1}^{k}$ for $k \geq 1$. Thus we obtain a square-root singularity for the amplitude of the potential $\psi$ and for the density profile.

On the other hand, we can also obtain asymptotic expressions for the equilibrium density profile in the limit of low temperatures $T \rightarrow 0$. Thus, close to $\zeta=0$ where $\psi \rightarrow-\infty$ we can approximate $\mathcal{V}(\psi) \simeq \mathrm{e}^{-\psi}$ which yields from Eq. (15)

$\zeta \ll \frac{\ln \zeta_{L}}{\zeta_{L}}: \quad \zeta \simeq \int_{\psi_{-}}^{\psi} \frac{\mathrm{d} \psi^{\prime}}{\sqrt{2\left(\mathrm{e}^{\left.-\psi_{-}-\mathrm{e}^{-\psi^{\prime}}\right)}\right.}}$,

which leads to:

$\zeta \ll \frac{\ln \zeta_{L}}{\zeta_{L}}: \quad \eta(\zeta) \simeq \frac{\zeta_{L}^{2}}{2 \cosh ^{2}\left(\zeta_{L} \zeta / 2\right)}$.

As expected, we recover the equilibrium profile obtained by Camm (1950) for an isolated 1-D gravitational system. Indeed, close to the density peak we can neglect $\bar{\rho}$ in Poisson Eq. (11)

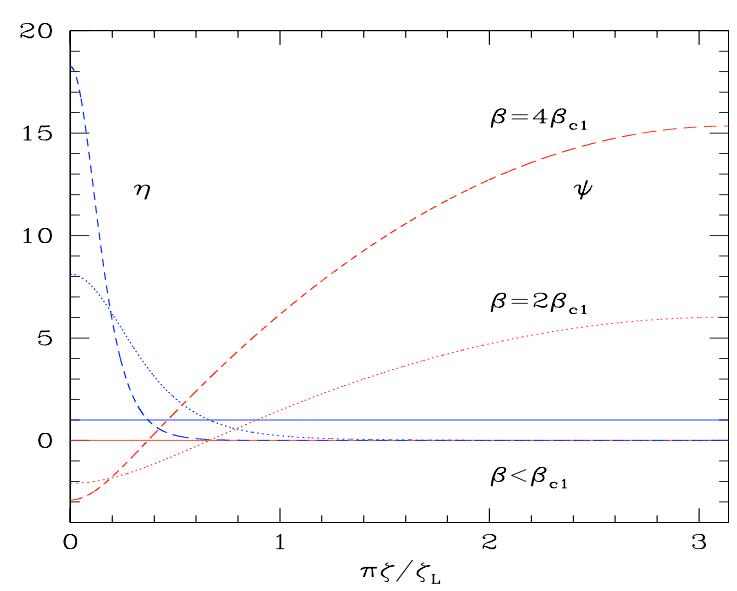

Fig. 1. The equilibrium profile $n=1$ obtained at $\beta=2 \beta_{c 1}$ (dotted lines) and $\beta=4 \beta_{c 1}$ (dashed lines). The solid lines correspond to the uniform solution $(n=0)$ with $\eta=1$ and $\psi=0$. The normalized density profiles $\eta$ are the curves with a maximum at $\zeta=0$ while the normalized potentials $\psi$ have a maximum at $\zeta=\zeta_{L}$.

in the low temperature limit (since $\rho(0) \sim 1 / T$ from Eq. (23)). Thus for arbitrary $n$ we obtain a series of density peaks which follow the equilibrium profile of isolated 1-D gravitational systems separated by low density regions since in the case of the mode $n=1$ we obtain:

$\eta\left(\zeta_{L}\right) \sim \mathrm{e}^{-\zeta_{L}^{2} / 2}=\mathrm{e}^{-\pi^{2} T_{c 1} / 2 T}$,

where we used the approximation $\mathcal{V}(\psi) \simeq \psi$ in the underdense region. We can note that the density in these underdense regions decreases very fast at low temperatures.

We display in Fig. 1 the equilibrium profiles $n=0$ (homogeneous solution) and $n=1$ (one density peak at $\zeta=0$ ) at temperatures $T_{c 1} / 2$ and $T_{c 1} / 4$. We can already check at these temperatures that the density peak grows as $1 / T$ while its width decreases as $T$ and that the underdense region is almost void. Of course, the potential $\psi=-\ln \eta$ shows a much shallower shape.

Thermodynamical quantities:

From Eq. (10) the distribution function at equilibrium is:

$f(x, v)=\rho(x) \sqrt{\frac{\beta}{2 \pi}} \mathrm{e}^{-\beta v^{2} / 2}$,

whence the entropy $S$ defined in Eq. (8) is up to an additional constant:

$S=\frac{M}{2} \ln T-\int \mathrm{d} x \rho \ln \frac{\rho}{\bar{\rho}}=\frac{M}{2} \ln T+\bar{\rho} \int \mathrm{d} x \eta \psi$,

where we used Eqs. (14). Therefore, the specific entropy $s$ normalized such that $s\left(T_{c 1}\right)=0$ is:

$s=\frac{S-S\left(T_{c 1}\right)}{M}=\frac{1}{2} \ln \frac{T}{T_{c 1}}+\int_{0}^{\zeta_{L}} \frac{\mathrm{d} \zeta}{\zeta_{L}} \eta \psi$.

On the other hand, the energy $E$ at thermodynamical equilibrium can be written from Eqs. (8) and (10) as:

$E=\frac{M T}{2}+\int \mathrm{d} x \rho \frac{\phi+V}{2}$ with $\rho=\bar{\rho}+\frac{1}{2 g} \frac{\mathrm{d}^{2} \phi}{\mathrm{d} x^{2}}$. 


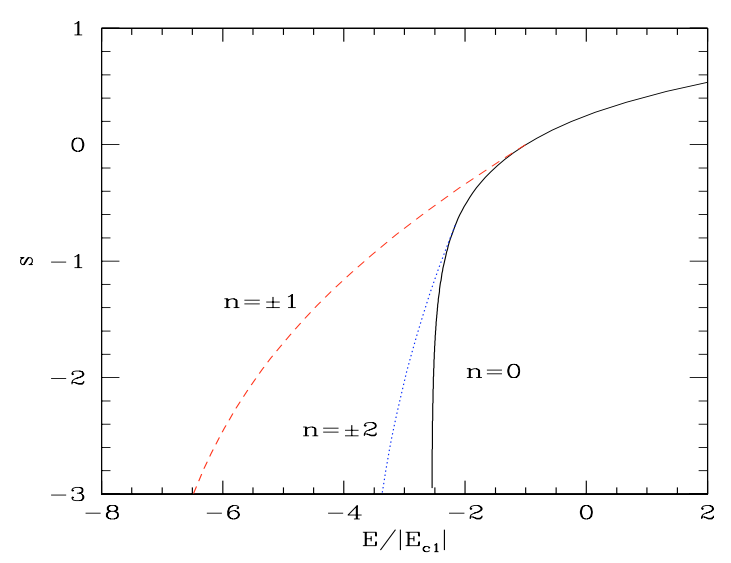

Fig. 2. The specific entropy $s=\left(S-S\left(T_{c 1}\right)\right) / M$ as a function of the energy $E$ for the modes $n=0, \pm 1$ and \pm 2 . The lower energy bound $E_{\min }(n)$ associated with mode $\pm n$, reached at $T=0$, increases for higher $n>0$. The homogeneous solution $n=0$ has the highest bound $E_{\mathrm{H}}$. All thermodynamical quantities are identical for both equilibria $n$ and $-n$.

Integrating over the term $\rho V$, using the second Eq. (28) and integration by parts, yields:

$E=\frac{M T}{2}-\frac{g M^{2} L}{6}+\frac{M}{4}[\phi(0)+\phi(L)]+\int \mathrm{d} x(\rho-\bar{\rho}) \frac{\phi}{2}$.

From Eq. (5) we obtain $\phi(0)+\phi(L)=0$ therefore the energy of modes 0 and $\pm n$ reads:

$$
\begin{aligned}
E_{0} & =\frac{M T}{2}-\frac{g M^{2} L}{6} \\
E_{ \pm n} & =\frac{M T}{2}-\frac{g M^{2} L}{6}+\frac{M T}{2} \int_{0}^{\zeta_{L}} \frac{\mathrm{d} \zeta}{\zeta_{L}}(\eta-1) \psi .
\end{aligned}
$$

In particular, the energy $E_{c n}$ associated with the critical temperature $T_{c n}$ of Eq. (17) is:

$E_{c n}=-\frac{n^{2} \pi^{2}-6}{6 n^{2} \pi^{2}} g M^{2} L \quad$ at $\quad T_{c n}=\frac{2 g M L}{n^{2} \pi^{2}}$.

Here we can note that the energy $E$ of the OSC system is actually bounded from below. Indeed, as can be clearly seen from Eq. (7) the minimum value $E_{\min }$ for the energy is reached when all the mass is at rest at $x=0$ or $x=L$. On the other hand the energy $E_{\mathrm{H}}$ associated with the homogeneous static solution $\rho=\bar{\rho}$ and $v=0$, which would be the counterpart of the original Hubble flow, can be obtained from Eq. (30) with $T=0$. This yields:

$E_{\min }=-\frac{g M^{2} L}{2}$ and $E_{\mathrm{H}}=-\frac{g M^{2} L}{6}$.

We can note that since $E_{\mathrm{H}}=E_{0}(T=0)$ all equilibrium solutions $\pm n$ can be reached with this energy, as may also be seen from Fig. 2 where we display the specific entropy $s$ as a function of the energy $E$. As we can check in Fig. 2 the mode $n= \pm 1$ reaches the energy bound $E_{\min }$ at $T=0$ while other equilibrium states $\pm n$ are increasingly close for larger $n$ to the homogeneous solution, with respect to global quantities such as energy or entropy, since they are made of $n / 2$ density peaks separated by a length $2 \zeta_{L} / n$, and their minimal energy $E_{\min }(n)$ gets closer to $E_{\mathrm{H}}$. Although Fig. 2 only shows the

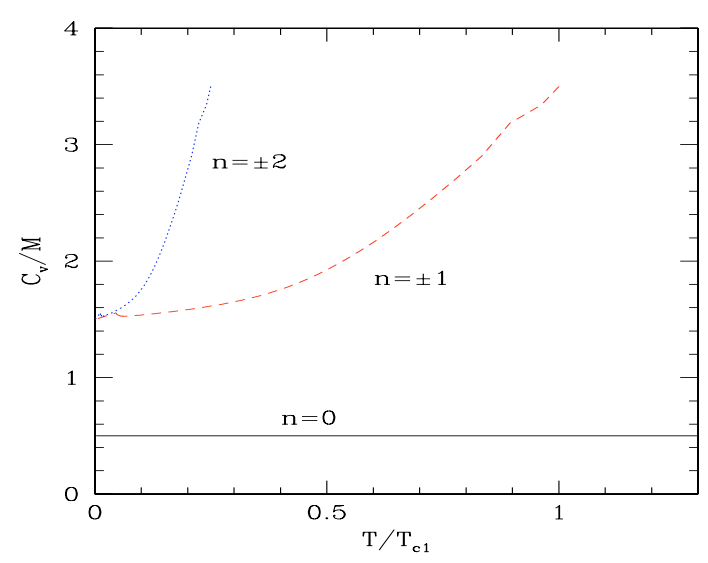

Fig. 3. The specific heat $C_{v}=\mathrm{d} E / \mathrm{d} T$ as a function of temperature for the equilibria $n=0, \pm 1$ and \pm 2 . It is discontinuous at the transitions $T_{c n}$ and corresponds to a second-order phase transition.

modes $0, \pm 1$ and \pm 2 we can see that at a fixed energy $E$ the homogeneous state has the lowest entropy while modes $\pm n$ with $n \geq 1$ have a higher entropy as $n$ decreases. In particular, below $T_{c 1}$ the mode \pm 1 with only one density peak at $x=0$ or $x=L$ is the highest entropy equilibrium. Therefore, it is a stable equilibrium solution of the system, as we shall also check in Sect. 3.2.1, whereas the homogeneous solution is unstable. Thus, as in the case of isolated 1-D systems (where only equilibrium \pm 1 exists and extends to all temperatures) and contrary to 3-D systems we have no gravothermal catastrophe (associated with the disappearance of any local entropy maximum, e.g. Binney \& Tremaine 1987; Padmanabhan 1990).

Of course, the energy $E$ is continuous at the transitions $T_{c n}$. However, its derivative $C_{v}=\mathrm{d} E / \mathrm{d} T$ is discontinuous as we jump onto the branch $\pm n$. Indeed, from Eq. (30) the specific heat $C_{v 0}$ associated with the uniform solution is:

$C_{v 0}=\frac{M}{2}$

while near the transition $T_{c 1}$, using the expansion (20)-(21), we obtain:

$E_{1} \simeq E_{c 1}+\frac{7 M}{2}\left(T-T_{c 1}\right)$ hence $C_{v 1}\left(T_{c 1}^{-}\right)=\frac{7 M}{2}$

We show in Fig. 3 the specific heat for $n=0, \pm 1$ and \pm 2 . We can check the discontinuity at $T_{c n}$ which corresponds to a second-order phase transitions. Next, we display in Fig. 4 the calorific curve $\beta(E)$. It also clearly exhibits this secondorder phase transition and we can see on the figure the various low energy bounds $E_{\min }(n)$. Besides, we can see in Fig. 4 that the specific heat is always positive and no gravothermal catastrophe can develop. Overall, the behaviour of the OSC model is quite similar to the HMF model studied in details in Chavanis et al. (2005). The latter model is defined by a cosine self-interaction $\cos \left[2 \pi\left(x_{i}-x_{j}\right) / L\right]$ instead of $\left|x_{i}-x_{j}\right|$ without the "external" potential $V$. It can also be seen as a truncation of the 1-D gravitational potential $\Phi$ to its first Fourier component. As described in Chavanis et al. (2005) the HMF model also exhibits a second-order phase transition from an homogeneous equilibrium above $T_{c 1}$ to a clustered phase with only one density peak below $T_{c 1}$. However, there are no other (unstable) 


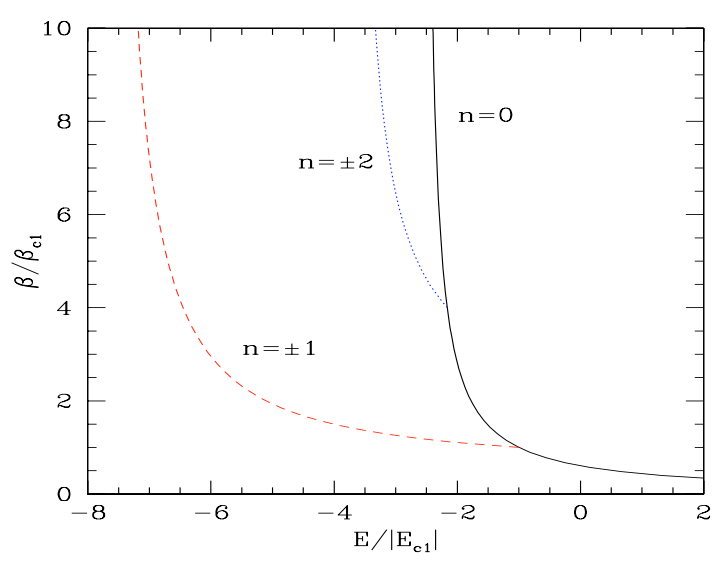

Fig. 4. The calorific curve $\beta(E)$ for the modes $n=0, \pm 1$ and \pm 2 . It shows a second-order phase transition at $T_{c 1}$.

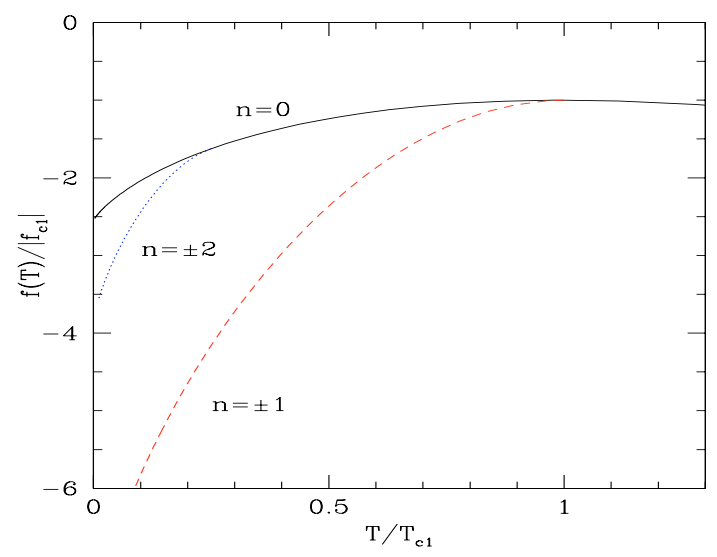

Fig. 5. The free energy $f(T)=F(T)+T S\left(T_{c 1}\right)$ as a function of temperature for the states $n=0, \pm 1$ and \pm 2 .

equilibria $\pm n$ appearing at $T_{c n}$. Of course, the infinite series of equilibria $\pm n$ which appear in the gravitational case is due to the scale-free nature of the gravitational potential $\propto\left|x_{i}-x_{j}\right|$ (which is only broken by the finite length $L$ of the system which leads to a discrete spectrum instead of a continuous one). We shall check in the following that apart for the additional equilibria $\pm n$ the properties of the HMF system and of the OSC model (7) are similar both from thermodynamical and dynamical points of view.

\subsubsection{Canonical ensemble}

In the canonical ensemble the thermodynamical equilibrium is obtained by minimizing the free energy $F=E-T S$ at fixed temperature $T$ and mass $M$. This yields again the MaxwellBoltzmann distribution (10) hence we recover the same critical temperatures and solutions (17)-(21). The free energy $F(T)$ reads:

$$
\begin{aligned}
F+T S\left(T_{c 1}\right)= & E-M T s=\frac{M T}{2}-\frac{g M^{2} L}{6} \\
& -\frac{M T}{2} \ln \left(\frac{T}{T_{c 1}}\right)-\frac{M T}{2} \int_{0}^{\zeta_{L}} \frac{\mathrm{d} \zeta}{\zeta_{L}}(\eta+1) \psi
\end{aligned}
$$

We display in Fig. 5 the free energy $F$ as $F(T)+T S\left(T_{c 1}\right)$ as a function of temperature for equilibria $n=0, \pm 1$ and \pm 2 . We can see that at fixed $T$ the homogeneous state $(n=0)$ has the highest free energy while $F$ decreases for lower $n$ with $n \geq 1$. Therefore, as we shall check in Sect. 3.2.2 the uniform equilibrium is again unstable below $T_{c 1}$ while the equilibrium $n= \pm 1$ is stable.

\subsubsection{Grand-canonical ensemble}

In the grand-canonical ensemble the thermodynamical equilibrium is obtained by minimizing the grand potential $\Omega=$ $E-T S-\mu M$ at fixed temperature $T$ and chemical potential $\mu$. Since the mass $M$ now fluctuates the mean density $\langle\rho\rangle=M / L$ within $[0, L]$ is no longer set equal to $\bar{\rho}$. In this case, we cannot any longer extend the system to the whole real line using a periodicity of $2 L$ and symmetry with respect to $x=0$. It can only be interpreted as a small system of actual size $L$ within a larger cosmological background of mean density $\bar{\rho}$, analyzed over time-scales much smaller than the Hubble time. Thus, if $\langle\rho\rangle>\bar{\rho}$ it corresponds to a local overdensity while if $\langle\rho\rangle\langle\bar{\rho}$ it corresponds to a local underdensity. Then, minimizing the grand potential $\Omega$ yields the same results (17)-(21) as for the micro-canonical and canonical ensembles. Note that the agreement between all three thermodynamical ensembles does not hold for all 1-D systems. For instance a system of concentric spherical cells which interact by gravity can show metastable states, negative specific heat and discrepancies between different thermodynamical ensembles as seen in Youngkins \& Miller (2000). Thus the 1-D system studied here from planar perturbations shows a rather simple behaviour while presenting a phasetransition and a scale-free origin.

\subsection{Thermodynamical stability}

\subsubsection{Micro-canonical ensemble}

As recalled in Sect. 3.1.1 the thermodynamical equilibrium states within the micro-canonical ensemble are obtained by maximizing the entropy $S$. In Sect. 3.1.1 we investigated the first variations of the entropy $S$, which yields maxima, minima and saddle-points. Next, the thermodynamical stability of these states is obtained by studying the second variations of the entropy. A stable equilibrium state corresponds to negative definite second variations. Following Padmanabhan (1990), maximizing the entropy $S[f]$ at fixed energy $E$ and density $\rho(x)$ yields the Maxwellian distribution (25). Then, we can write the entropy $S$ and the energy $E$ in terms of the density $\rho(x)$ as in Eqs. (26), (28). Next, we can obtain from these expressions the second variation $\delta^{2} S$ with respect to a density fluctuation $\delta \rho(x)$ at fixed energy (the constraint $\delta E=0$ yields $\delta T$ in terms of $\delta \rho$, which is next substituted into $\delta S$ ). This yields for the second variation around an equilibrium solution:

$$
\begin{aligned}
\delta^{2} S= & -\frac{g}{2 T} \int \mathrm{d} x \mathrm{~d} x^{\prime} \delta \rho(x) \delta \rho\left(x^{\prime}\right)\left|x-x^{\prime}\right|-\int \mathrm{d} x \frac{\delta \rho^{2}}{2 \rho} \\
& -\frac{1}{M T^{2}}\left(\int \mathrm{d} x \phi \delta \rho\right)^{2} .
\end{aligned}
$$


As in Padmanabhan (1990) let us define the mass fluctuation $q(x)$ below $x$ as:

$\delta \rho=\frac{\mathrm{d} q}{\mathrm{~d} x}, \quad q(x)=\int_{0}^{x} \mathrm{~d} x^{\prime} \delta \rho\left(x^{\prime}\right), \quad q(0)=q(L)=0$,

where we used the conservation of mass $M$. Then, after integrations by parts the second variation $\delta^{2} S$ can be written as:

$\delta^{2} S=\int_{0}^{L} \mathrm{~d} x \mathrm{~d} x^{\prime} q(x) K_{M C}\left(x, x^{\prime}\right) q\left(x^{\prime}\right)$

with:

$$
\begin{aligned}
K_{M C}\left(x, x^{\prime}\right)= & \frac{g}{T} \delta_{D}\left(x-x^{\prime}\right)-\frac{1}{M T^{2}} \frac{\mathrm{d} \phi}{\mathrm{d} x}(x) \frac{\mathrm{d} \phi}{\mathrm{d} x}\left(x^{\prime}\right) \\
& +\frac{1}{2} \delta_{D}\left(x-x^{\prime}\right) \frac{\mathrm{d}}{\mathrm{d} x^{\prime}}\left(\frac{1}{\rho\left(x^{\prime}\right)} \frac{\mathrm{d}}{\mathrm{d} x^{\prime}}\right)
\end{aligned}
$$

Thus, the equilibrium $\rho(x)$ is stable if all eigenvalues $\lambda$ of the kernel $K_{M C}$ are negative (so that $S[\rho]$ is a maximum). Hence we are led to investigate the eigenvalue problem $K_{M C} \cdot q=\lambda q$ which reads:

$\frac{1}{2} \frac{\mathrm{d}}{\mathrm{d} x}\left[\frac{1}{\rho(x)} \frac{\mathrm{d} q}{\mathrm{~d} x}\right]+\frac{g}{T} q(x)=\lambda q(x)+\frac{\phi^{\prime}(x)}{M T^{2}} \int \mathrm{d} x^{\prime} \phi^{\prime} q$.

In terms of the dimensionless variables (14) we obtain the eigenvalue problem:

$\frac{\mathrm{d}}{\mathrm{d} \zeta}\left[\frac{1}{\eta} \frac{\mathrm{d} q}{\mathrm{~d} \zeta}\right]+q=\tilde{\lambda} q+2 \psi^{\prime} \int \frac{\mathrm{d} \zeta^{\prime}}{\zeta_{L}} \psi^{\prime} q$, with $\tilde{\lambda}=\frac{\lambda}{g \beta}$,

supplemented by the boundary conditions $q(0)=q\left(\zeta_{L}\right)=0$. We can note that the matrix $\tilde{K}_{M C}$ defined by:

$\tilde{K}_{M C} \cdot q=-\frac{\mathrm{d}}{\mathrm{d} \zeta}\left[\frac{1}{\eta} \frac{\mathrm{d} q}{\mathrm{~d} \zeta}\right]+2 \psi^{\prime} \int \frac{\mathrm{d} \zeta^{\prime}}{\zeta_{L}} \psi^{\prime} q$

is positive definite, therefore $\tilde{\lambda} \leq 1$.

For the homogeneous equilibrium $(n=0)$ where $\eta=1$ and $\psi=0$ we obtain:

$q^{\prime \prime}+(1-\tilde{\lambda}) q=0, \quad q(0)=q\left(\zeta_{L}\right)=0$,

whose solutions are:

$q_{k}=A_{k} \sin \left(k \pi \frac{\zeta}{\zeta_{L}}\right), \quad \tilde{\lambda}_{k}=1-\left(\frac{k \pi}{\zeta_{L}}\right)^{2}, \quad k=1,2, \ldots$

Thus, as expected we find that the uniform equilibrium is stable at high temperature $T>T_{c 1}$ (i.e. $\zeta_{L}<\pi$ ). At each critical temperature $T_{c n}$ defined in Eq. (17) a new mode of instability $\lambda_{n}$ appears while two new equilibrium states $\pm n$ appear which exhibit $n-1$ unstable modes. This agrees with Fig. 2 which displays the specific entropy as a function of energy. In particular, close to $T_{c 1}^{-}$we can investigate the stability of equilibrium $n= \pm 1$ by using the expansion (20)-(21) and looking for a similar expansion for $\lambda_{1}$. Thus, we write:

$q_{1}=q_{1,0}+a_{1} q_{1,1}+a_{1}^{2} q_{1,2}+\ldots, \quad \tilde{\lambda}_{1}=a_{1}^{2} \tilde{\lambda}_{1,2}+\ldots$

which yields (we can check that $\tilde{\lambda}_{1,1}=0$ ):

$q_{1,0}=\sin \zeta, q_{1,1}=B_{1} \sin \zeta-\frac{1}{3} \sin 2 \zeta, \quad \tilde{\lambda}_{1,2}=-\frac{5}{4}$.

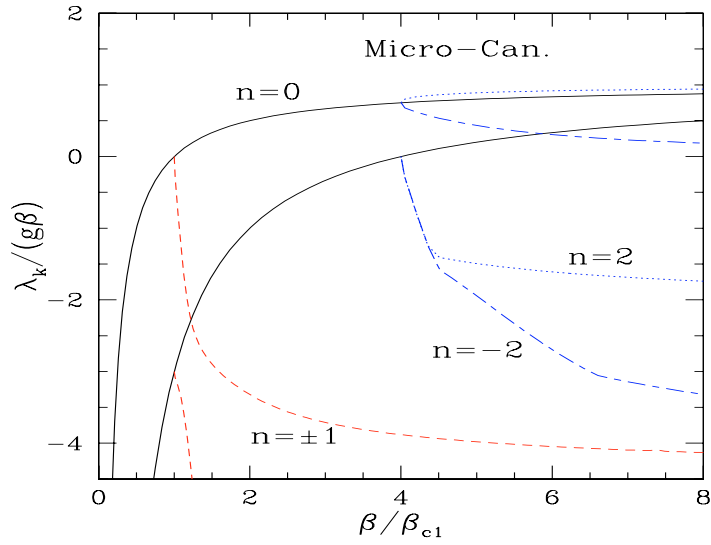

Fig. 6. The largest two stability eigenvalues $\tilde{\lambda}_{k}=\lambda_{k} / g \beta$ (i.e. $\left.k=1,2\right)$ as a function of inverse temperature $\beta$ for the equilibrium states 0 (solid lines), \pm 1 (dashed lines), 2 (dotted lines) and -2 (dot-dashed lines) for the micro-canonical ensemble. Stability corresponds to negative eigenvalues $\tilde{\lambda}_{k}<0$. The states \pm 1 are actually identical (up to a symmetry with respect to $x=L / 2$ ). The equilibria $\pm n$ show $n-1$ unstable eigenvalues while the homogeneous solution $n=0$ shows $n$ unstable eigenvalues below $T_{c n}$.

Therefore, since $\lambda_{1}<0$ close to $T_{c 1}^{-}$we see that equilibria \pm 1 are stable close to $T_{c 1}^{-}$while equilibrium 0 becomes unstable. From Fig. 2 we see that the states \pm 1 are actually stable down to $T=0$. We display in Fig. 6 the first two stability eigenvalues $\tilde{\lambda}_{k}$ as a function of $\beta$ for the equilibrium solutions $n=0, \pm 1$ and \pm 2 , obtained from a numerical study of Eq. (42). We can check that the equilibria \pm 1 are stable above $\beta_{c 1}$ while the homogeneous equilibrium is unstable. Higher states $\pm n$ exhibit $n-1$ unstable eigenvalues therefore they are only saddle-points of the entropy $S[f]$ and they are not thermodynamically stable.

The series of equilibrium states $\pm n$ which appear as new unstable modes from the homogeneous solution at increasingly low $T_{c n}$ are somewhat analogous to the high order modes of instability which appear for a 3-D finite isothermal sphere obtained in Semelin et al. (1999) and Chavanis (2002). They can be related to the scale-free nature of gravitational interactions (although the homogeneous background yields an arithmetic hierarchy of scales while the isothermal sphere leads to a geometric progression). However, since the equilibria $\pm n$ with $n \geq 2$ are unstable (except $n=2$ at low $T$ for a collisionless dynamics as seen in Sect. 5 below) a discrete $N$-body system which undergoes collisional relaxation is not expected to fragment into many clumps at low $T$ but to form only one collapsed density peak close to one boundary (though several temporary clumps might appear in a transient regime).

\subsubsection{Canonical ensemble}

Within the canonical ensemble we need to minimize the free energy $F$. Hence stable equilibria are characterized by negative definite second variations of $-F$ at fixed temperature and mass. Following the same procedure as in Sect. 3.2.1 we obtain a similar eigenvalue problem with a kernel $K_{\mathrm{C}}\left(x, x^{\prime}\right)$ which is equal to $K_{\mathrm{MC}}\left(x, x^{\prime}\right)$ written in Eq. (40) without the 


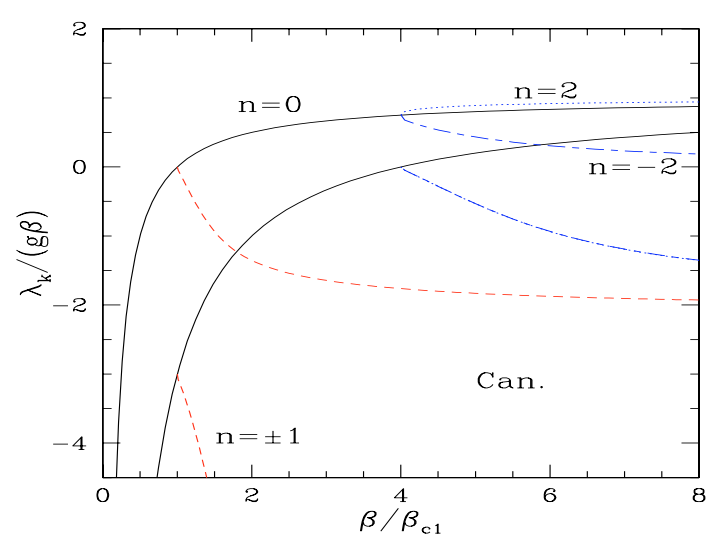

Fig. 7. The stability eigenvalues $\tilde{\lambda}_{k}=\lambda_{k} / g \beta$ as a function of inverse temperature $\beta$ for the equilibria $0, \pm 1$ and \pm 2 for the canonical ensemble. Stability corresponds to negative eigenvalues $\tilde{\lambda}_{k}<0$.

term $\phi^{\prime}(x) \phi^{\prime}\left(x^{\prime}\right) / M T^{2}$. Therefore, the eigenvalue problem now reads:

$\frac{\mathrm{d}}{\mathrm{d} \zeta}\left[\frac{1}{\eta} \frac{\mathrm{d} q}{\mathrm{~d} \zeta}\right]+q=\tilde{\lambda} q, \quad q(0)=q\left(\zeta_{L}\right)=0$.

We again obtain as in Eq. (43) that $\tilde{\lambda} \leq 1$. Then, the stability properties of the uniform solution are exactly the same as for the micro-canonical ensemble (since $\psi=0$ ). On the other hand, for equilibrium 1 we can again use the expansion (20)-(21) close to $T_{c 1}^{-}$. This now yields $\tilde{\lambda}_{1,2}=-1 / 4$. Therefore, states \pm 1 are again stable close to $T_{c 1}^{-}$. We display in Fig. 7 the stability eigenvalues obtained from a numerical study of Eq. (48). We obtain a behaviour which is similar to Fig. 6 and we recover the same thermodynamical stability properties. This agrees with Fig. 5 for the free energy. Note that the equivalence between the micro-canonical and the canonical ensemble (with, however, different eigenvalues) obtained in this case is similar to that found for the HMF model but differs from the case of 3-D self-gravitating systems where the limits of stability do not coincide in micro-canonical and canonical ensembles (Padmanabhan 1990; Chavanis 2002). This is also related to the fact that the specific heat is always positive (see Fig. 3) which is not the case for 3-D self-gravitating systems (in the micro-canonical ensemble).

\subsubsection{Grand-canonical ensemble}

Within the grand-canonical ensemble we now need to minimize the grand potential $\Omega$. The second variation of $\Omega$ reads:

$\delta^{2} \Omega=\frac{g}{2} \int \mathrm{d} x \mathrm{~d} x^{\prime} \delta \rho(x) \delta \rho\left(x^{\prime}\right)\left|x-x^{\prime}\right|+T \int \mathrm{d} x \frac{\delta \rho^{2}}{2 \rho}$.

Since the total mass is now allowed to fluctuate we define the perturbation $\delta \bar{\rho}$ of the mean density $M / L$ and $q(x)$ by:

$\delta \rho(x)=\delta \bar{\rho}+q^{\prime}(x), \quad q(0)=q(L)=0$.

Substituting into Eq. (49) and integrating by parts yields:

$$
\begin{aligned}
\delta^{2} \Omega= & -g \int \mathrm{d} x q^{2}-\frac{T}{2} \int \mathrm{d} x q \frac{\mathrm{d}}{\mathrm{d} x}\left(\frac{q^{\prime}}{\rho}\right)+\frac{\delta \bar{\rho}^{2}}{2} \frac{g L^{3}}{3} \\
& +\frac{\delta \bar{\rho}^{2}}{2} T \int \frac{\mathrm{d} x}{\rho}-\delta \bar{\rho} \int \mathrm{d} x q\left[-T \frac{\rho^{\prime}}{\rho^{2}}+g(2 x-L)\right] .
\end{aligned}
$$

Equation (51) shows that $\delta^{2} \Omega$ has a finite minimum over $\delta \bar{\rho}$. Thus, we first minimize $\delta^{2} \Omega$ over $\delta \bar{\rho}$ and the second variation of $\Omega$ with respect to $q(x)$ alone now reads:

$$
\begin{aligned}
& -\frac{\delta^{2} \Omega}{T}(q)=\frac{1}{2} \int \mathrm{d} x q \frac{\mathrm{d}}{\mathrm{d} x}\left(\frac{q^{\prime}}{\rho}\right)+\frac{g}{T} \int \mathrm{d} x q^{2} \\
& +\left(\frac{g L^{3}}{3 T}+\int \frac{\mathrm{d} x}{\rho}\right)^{-1}\left[\int \mathrm{d} x q\left[\frac{g}{T}\left(x-\frac{L}{2}\right)-\frac{\rho^{\prime}}{2 \rho^{2}}\right]\right]^{2} .
\end{aligned}
$$

This can again be written in terms of a kernel $K_{\mathrm{GC}}\left(x, x^{\prime}\right)$ which leads to the eigenvalue problem:

$$
\begin{aligned}
\frac{\mathrm{d}}{\mathrm{d} \zeta}\left[\frac{1}{\eta} \frac{\mathrm{d} q}{\mathrm{~d} \zeta}\right]+q= & \tilde{\lambda} q-\left(\frac{\zeta_{L}^{3}}{6}+\int \frac{\mathrm{d} \zeta}{\eta}\right)^{-1} \\
& \times\left(\frac{\mathrm{d}}{\mathrm{d} \zeta}\left(\frac{1}{\eta}\right)+\left(\zeta-\frac{\zeta_{L}}{2}\right)\right) \\
& \times \int \mathrm{d} \zeta^{\prime}\left(\frac{\mathrm{d}}{\mathrm{d} \zeta^{\prime}}\left(\frac{1}{\eta}\right)+\left(\zeta^{\prime}-\frac{\zeta_{L}}{2}\right)\right) q\left(\zeta^{\prime}\right)
\end{aligned}
$$

For the uniform solution this equation reads:

$$
q^{\prime \prime}+(1-\tilde{\lambda}) q=-\frac{\zeta-\frac{\zeta_{L}}{2}}{\zeta_{L}+\frac{\zeta_{L}^{3}}{6}} \int \mathrm{d} \zeta^{\prime}\left(\zeta^{\prime}-\frac{\zeta_{L}}{2}\right) q\left(\zeta^{\prime}\right)
$$

For odd $k$ we recover the modes (45) of the micro-canonical and canonical ensembles (since the r.h.s. in Eq. (54) vanishes) whereas even modes are modified. In particular, their eigenvalues are now given by:

$\theta \cot \theta=1-\frac{\theta^{2}}{3}-\theta^{4}\left(\frac{16}{\zeta_{L}^{4}}+\frac{8}{3 \zeta_{L}^{2}}\right)$ with $\tilde{\lambda}=1-\left(\frac{2 \theta}{\zeta_{L}}\right)^{2}$.

For large $\theta$ this yields $\theta_{2 k} \simeq k \pi$ hence we recover the even modes $\tilde{\lambda}_{2 k} \simeq 1-\left(2 k \pi / \zeta_{L}\right)^{2}$ of (45). One can see from Eq. (55) that $\tilde{\lambda}$ is increased at fixed $\zeta_{L}$ as compared with the microcanonical and canonical result (45). We can note that for $\zeta_{L}>$ $\sqrt{60+12 \sqrt{30}}$ the first even mode has $\tilde{\lambda}_{2}>1$, that is $\theta_{2}$ is imaginary and can be written as $\theta_{2}=i \hat{\theta}_{2}$ with $\hat{\theta}_{2} \operatorname{coth} \hat{\theta}_{2}=$ $1+\hat{\theta}_{2}^{2} / 3-\hat{\theta}_{2}^{4}\left(16 / \zeta_{L}^{4}+8 / 3 \zeta_{L}^{2}\right)$. In any case, we find that as for the micro-canonical and canonical ensembles the homogeneous equilibrium is unstable below $T_{c 1}$ while other equilibrium solutions $\pm n$ are also unstable except for $n=1$, as can be seen from Fig. 8 where we show the results of a numerical analysis of the eigenvalue problem (53). Again, it is possible to analyze the stability of state \pm 1 near the transition $T_{c 1}$ using the expansion (20)-(21). This yields $\tilde{\lambda}_{1,2}=-1 / 4<0$ which implies that equilibria \pm 1 are again stable close to $T_{c 1}^{-}$. From Fig. 8 we see they are actually stable down to $T=0$. Thus, all three thermodynamical ensembles lead to the same stability properties.

\section{Hydrodynamical model}

The thermodynamical analysis of Sect. 3 has allowed us to obtain the equilibrium states of the OSC system (7) and to derive their thermodynamical stability properties. However, this analysis does not yield the growth rates associated with such instabilities nor the dynamical properties of the system. Within 


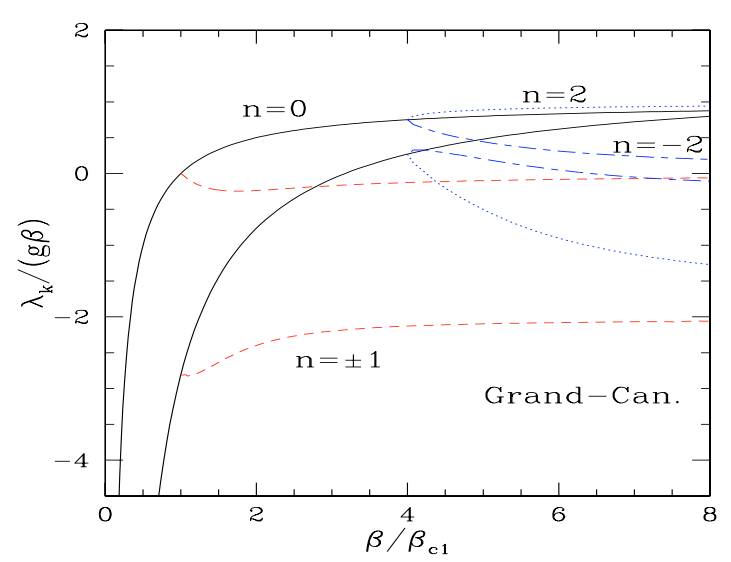

Fig. 8. The stability eigenvalues $\tilde{\lambda}_{k}=\lambda_{k} / g \beta$ as a function of inverse temperature $\beta$ for the equilibria $0, \pm 1$ and \pm 2 for the grand-canonical ensemble. Stability corresponds to negative eigenvalues $\tilde{\lambda}_{k}<0$.

a mean field or continuum approach we shall study in Sect. 5 the dynamics as given by the Vlasov-Poisson system. However, before we tackle the Vlasov equation in phase-space $(x, v)$ we first investigate in this section the hydrodynamical model associated with the hamiltonian (7). Indeed, the fluid and collisionless systems often share important properties while the hydrodynamical system is much easier to analyze (e.g. Binney \& Tremaine 1987).

\subsection{Hydrostatic equilibrium}

The equations of motion of the hydrodynamical system associated with the OSC model (7) are simply:

$\frac{\partial \rho}{\partial t}+\frac{\partial}{\partial x}(\rho v)=0$

$\frac{\partial v}{\partial t}+v \frac{\partial v}{\partial x}=-\frac{1}{\rho} \frac{\partial P}{\partial x}-\frac{\partial \phi}{\partial x}$,

where the potential $\phi$ is given by Eq. (6) and $P(\rho)$ is the barotropic pressure of the gas. As usual, one can check that the energy functional $W$ is conserved by the equations of motion (Binney \& Tremaine 1987), with:

$W=\int \mathrm{d} x \rho(x)\left[\int_{0}^{\rho} \mathrm{d} \rho^{\prime} \frac{P\left(\rho^{\prime}\right)}{\rho^{\prime 2}}\right]+\int \mathrm{d} x \rho \frac{v^{2}+\phi+V}{2}$.

Therefore, minima of $W$ are stationary solutions of the Euler equations which are non-linearly stable. As in the statistical analysis of Sect. 3.1 the first variation of $W$ yields the properties of such stationary states (in addition to saddle-points of $W$ ). Then, the first variation of $W$ with respect to $v$ leads to $v=0$. Substituting into the Euler Eq. (57) yields the hydrostatic equilibrium condition:

$\frac{\mathrm{d} P}{\mathrm{~d} x}=-\rho \frac{\mathrm{d} \phi}{\mathrm{d} x}$.

In the following we shall restrict ourselves to the case of the isothermal gas defined by the equation of state $P=\rho T$. Then Eq. (59) reads $\mathrm{d} \rho / \mathrm{d} x=-\beta \rho \mathrm{d} \phi / \mathrm{d} x$ which yields again $\rho \propto \mathrm{e}^{-\beta \phi}$ as in the thermodynamical approach (10). Therefore, we recover the same stationary states as in Sect. 3.1.

\subsection{Non-linear stability}

The equilibrium states obtained in Sect. 4.1 are non-linearly stable if they are minima of the energy functional $W$, that is if the second variations of $W$ are definite positive. The second variation with respect to $v$ is clearly positive from the expression (58) while $\delta^{2} W / \delta \rho \delta v=0$ since $v=0$ for equilibrium states. Therefore, we only need to study the second variation with respect to the density. For the isothermal gas this yields:

$\delta^{2} W=\frac{1}{2} \int \mathrm{d} x \delta \rho \delta \Phi+\frac{T}{2} \int \mathrm{d} x \frac{\delta \rho^{2}}{\rho}$.

This is the same equation as the second variation of the free energy $F$ obtained for the canonical ensemble in Sect. 3.1.2. Hence the non-linear stability properties of various equilibrium states are the same as those derived for the canonical ensemble. Therefore, the non-linear hydrodynamical stability properties are identical to the thermodynamical ones. This implies in particular from Eq. (45) that the homogeneous equilibrium is unstable below $T_{c 1}$ while equilibria $\pm n$ are unstable close to $T_{c n}$ for $n \geq 2$. From Fig. 7 they remain unstable down to $T \rightarrow 0$ while equilibrium \pm 1 is stable below $T_{c 1}$.

\subsection{Dynamical linear stability}

The analysis of Sect. 4.2 allowed us to discriminate between stable and unstable states but it does not provide the growth rates of unstable perturbations. To this order we need to investigate the dynamical linear stability of such equilibrium states. Thus, we linearize the equations of motion (56), (57) and we study the normal modes of the form $\delta \rho \sim \mathrm{e}^{\lambda t}$. Introducing again the mass fluctuation $q(x)$ as in Eq. (38) and using the dimensionless variables (14) we obtain the eigenvalue problem:

$\frac{\mathrm{d}}{\mathrm{d} \zeta}\left[\frac{1}{\eta} \frac{\mathrm{d} q}{\mathrm{~d} \zeta}\right]+q=\nu \frac{q}{\eta}$ with $v=\frac{\lambda^{2}}{2 g \bar{\rho}}$ and $\delta v=-\lambda \frac{q}{\rho}$

Thus, eigenmodes with $v<0$ (i.e. $\lambda$ is imaginary) are stable while eigenmodes with $v>0$ (i.e. $\lambda$ is real) are unstable. More precisely, for $v>0$ we have both a decaying and a growing mode $(\lambda= \pm \sqrt{v 2 g \bar{\rho}})$ distinguished by opposite velocity fields, as seen from the last equation in (61). At the point of marginal stability $v=0$ we recover the eigenvalue problem (48) of the canonical ensemble. Therefore, from Sect. 4.2 we see that the conditions of non-linear and linear stability are identical and also coincide with the canonical ensemble. Note that this equivalence also holds for the HMF model (Chavanis et al. 2005) as well as for some more general models (Chavanis 2006).

For the uniform equilibrium $(n=0)$ we have $\eta=1$ so that we recover the eigenvalue problem (44) of both the micro-canonical and canonical ensembles with the same solutions (45). Thus we find again that the homogeneous solution is unstable below $T_{c 1}$. For the equilibrium state \pm 1 we can again use the expansion (20)-(21) close to $T_{c 1}$ which yields $v_{1} \simeq-a_{1}^{2} / 4<0$. Therefore the equilibrium \pm 1 is again stable close to $T_{c 1}$. From Fig. 9 which shows the eigenvalues $v_{k}$ as a function of inverse temperature $\beta$ we can check that the equilibrium \pm 1 is stable below $T_{c 1}$ down to $T=0$ whereas 


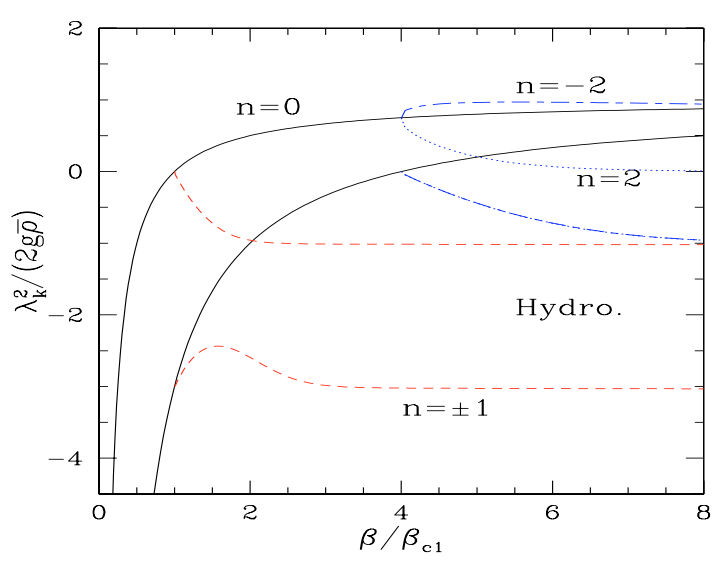

Fig. 9. The stability eigenvalues $v_{k}=\lambda_{k}^{2} /(2 g \bar{\rho})$ as a function of inverse temperature $\beta$ for the equilibria $0, \pm 1$ and \pm 2 for the hydrodynamical system. Stability corresponds to imaginary eigenvalues $\lambda_{k}^{2}<0$. The second eigenvalues $\lambda_{2}$ obtained for equilibria \pm 2 are equal.

equilibria $\pm n$ with $n \geq 2$ are always unstable. This is identical to the non-linear stability properties obtained in Sect. 4.2 and to the thermodynamical results of Sect. 3.2. We can note that the equilibria \pm 2 have different first eigenvalues $v_{1}>0$ but identical second eigenvalues $v_{2}<0$. Indeed, we can check numerically that this eigenmode vanishes both at the boundaries $x=0, L$, and at the midpoint $L / 2$ ( $q$ is antisymmetric with respect to $L / 2$ ) so that both $\eta$ and $\delta \eta$ are identical for $n=2$ and $n=-2$ if we extend them to the whole real axis up to a mere translation of $L / 2$. This is due to the symmetry properties of equilibrium profiles \pm 2 .

Next, we can note that the eigenvalue $v_{1}$ obtained for the state $n=-2$ quickly becomes very close to unity above $T_{c 2}$. In fact, we can check that $q=\eta$ is a solution of the differential Eq. (61) with $v=1$, using $\eta=\mathrm{e}^{-\psi}$ and $\psi^{\prime \prime}=\eta-1$ which characterize the equilibrium profiles, see Eqs. (14) and (15). Because of the boundary conditions $q(0)=q\left(\zeta_{L}\right)=0$ this is not an allowed solution to the eigenmode problem (61). However, for the equilibrium $n=-2$ where the overdensity goes to zero at the boundaries as $\eta \sim \mathrm{e}^{-\pi^{2} T_{c 2} / 2 T}$ from Eq. (24) the unstable eigenmode tends to $q \propto \eta$ at low $T$. From the definition (38) this implies for the density perturbation $\delta \eta \propto \eta^{\prime}(\zeta)$ which corresponds to a mere translation of the equilibrium density profile. We display in Fig. 10 the first eigenmode $v_{1} \simeq 1$ for the equilibrium state $n=-2$ at temperature $T_{c 2} / 6$. We plot the equilibrium profile $\eta$ (dashed line) and the growing mode $\delta \eta$ (solid line) with the associated velocity $\delta v$ (dotted line). The derivative $\eta^{\prime}(\zeta)$ normalized to the $\delta \eta$ at its peak cannot be distinguished from the perturbation $\delta \eta$ on the figure and we can see that the velocity is almost constant over the extent of the density peak. Thus the unstable mode obtained for the equilibrium $n=-2$ only corresponds to a translation of the density peak (which behaves as an isolated system as discussed in Sect. 3.1.1) while other eigenmodes $k \geq 2$ are decaying modes, as seen from Fig. 9. The associated eigenvalue $v_{1}=1$ merely corresponds to a point-particle which rolls down the "external" potential $V(x)$ of Eq. (6). Indeed, the solution of $\ddot{x}=-\mathrm{d} V / \mathrm{d} x$ reads $(x-L / 2) \propto \mathrm{e}^{ \pm \sqrt{2 g \bar{\rho}} t}$, which describes the motion in the low-temperature limit $T \rightarrow 0$ as the density peak (23) tends to

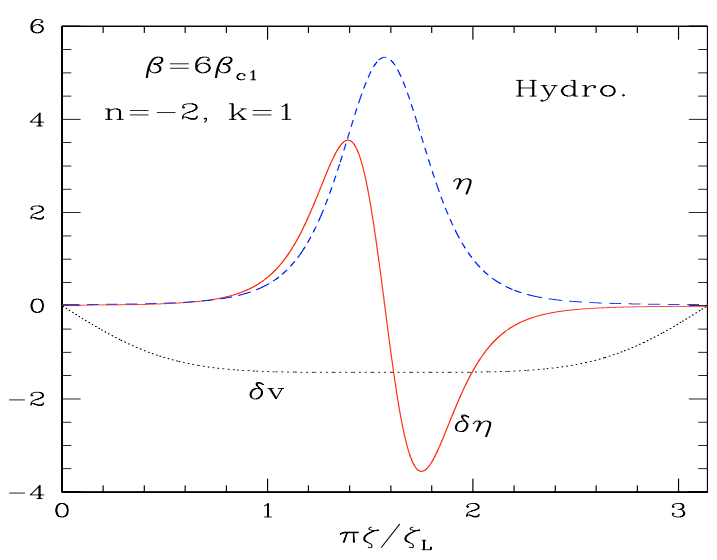

Fig. 10. The unstable eigenmode $k=1$ with $v_{1} \simeq 1$ for the equilibrium state $n=-2$ at temperature $\beta=6 \beta_{c 1}$. We plot the equilibrium density profile $\eta=\rho / \bar{\rho}$ (dashed line), the unstable eigenmode $\delta \eta=\delta \rho / \bar{\rho}$ (solid line) and the velocity $\delta v \propto-q / \eta$ (dotted line). This eigenmode corresponds to a mere translation of the density peak. The normalizations of $\delta \eta$ and $\delta v$ are arbitrary.

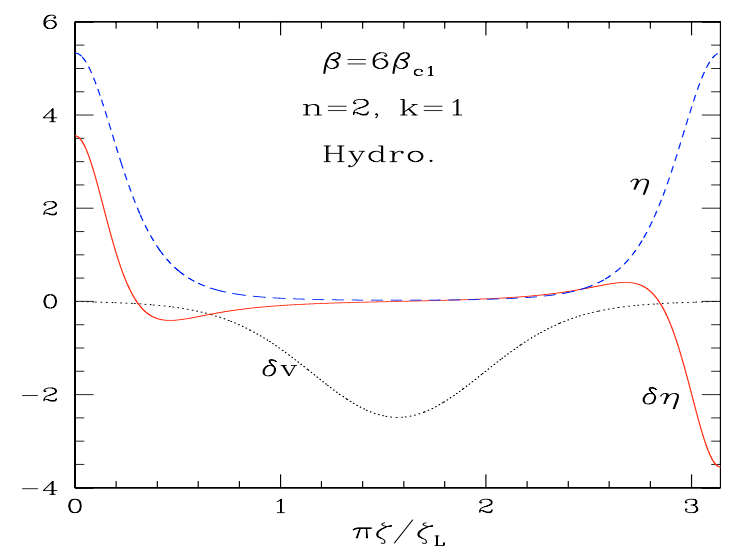

Fig. 11. The unstable eigenmode $k=1$ with $v_{1}>0$ for the equilibrium state $n=2$ at temperature $\beta=6 \beta_{c 1}$. We plot the equilibrium profile $\eta$ (dashed line), the unstable eigenmode $\delta \eta$ (solid line) and the velocity $\delta v$ (dotted line).

a Dirac. It would be interesting to follow the dynamics further from $x=L / 2$ to see whether the reflection at the boundaries $x=0, L$, quickly destroys the equilibrium profiles or whether some stationary cycle can be reached (if there were true solitonic solutions). We shall leave this analysis for future work as it goes beyond the scope of the present paper.

Finally, we can see in Fig. 9 that for the equilibrium $n=2$ the eigenvalue $v_{1}$ quickly goes to zero. Of course, we cannot recover the solution $q \propto \eta$ associated with a mere translation since the state $n=2$ is made of two density peaks at $x=0, L$, which grow at low $T$ so that the boundary conditions $q(0)=q(L)=0$ forbid the solution $q \propto \eta$. We show in Fig. 11 the first eigenmode $v_{1} \gtrsim 0$ for the equilibrium state $n=2$. It corresponds to a transfer of matter from one density peak towards the other one. The end-product of this process will be the equilibrium state \pm 1 which is the unique non-linearly stable equilibrium, as seen in Sect. 4.2. The growth rate of this instability quickly goes to zero at low $T$ as the underdense region which connects both density peaks reaches very low densities 
from Eq. (24) and the density peaks behave as almost isolated subsystems. Besides, it can be seen from the shape of $\delta \eta$ in Fig. 9 that as expected $\eta+\delta \eta$ corresponds to two unequal density peaks at $x=0, L$, which follow the profiles associated with isolated relaxed density peaks. Thus, in agreement with (23) we find that in the case displayed in Fig. 9 the peak at $x=0$ which gains some mass gets a higher density maximum and a smaller extension while the opposite holds for the peak at $x=L$ which looses mass. This can be checked more precisely from Eq. (61) as follows. The modified Lane-Emden Eq. (12) can also be written as:

$$
\frac{\mathrm{d}^{2}}{\mathrm{~d} \zeta^{2}}(-\ln \eta)=\eta-1, \text { whence } \frac{\eta^{\prime \prime}}{\eta}-\frac{\eta^{2}}{\eta^{2}}+\eta=1,
$$

which implies that if both $\eta$ and $\eta+\delta \eta$ are isothermal equilibrium solutions the difference $\delta \eta$ satisfies at first order:

$$
\frac{\delta \eta^{\prime \prime}}{\eta}-2 \frac{\eta^{\prime}}{\eta^{2}} \delta \eta^{\prime}+\left(1-\frac{\eta^{\prime \prime}}{\eta^{2}}+2 \frac{\eta^{\prime 2}}{\eta^{3}}\right) \delta \eta=0 .
$$

On the other hand, taking the derivative of the eigenmode Eq. (61) yields (with $q^{\prime}=\delta \eta$ ):

$$
\frac{\delta \eta^{\prime \prime}}{\eta}-2 \frac{\eta^{\prime}}{\eta^{2}} \delta \eta^{\prime}+\left(1-\frac{\eta^{\prime \prime}}{\eta^{2}}+2 \frac{\eta^{\prime 2}}{\eta^{3}}\right) \delta \eta=v\left(\frac{\delta \eta}{\eta}-\frac{\eta^{\prime}}{\eta^{2}} q\right) .
$$

Therefore, for $v=0$ we recover Eq. (63). In fact, it is clear that if $\left(\rho_{1}, v_{1}\right)$ and $\left(\rho_{2}, v_{2}\right)$ are two equilibrium solutions of the hydrodynamic Eqs. (56), (57) the difference $\left(\rho_{2}-\rho_{1}, v_{2}-v_{1}\right)$ is a static solution at linear order of the linearized equations of motion about $\left(\rho_{1}, v_{1}\right)$. Thus, in agreement with Fig. 9 the unstable eigenmode $v_{1} \rightarrow 0^{+}$obtained at low temperature for the equilibrium $n=2$ actually yields a quasi-static transfer of matter between the two density peaks in the sense that they follow equilibrium profiles (parameterized by their varying mass) at the fixed temperature $T$.

It can be checked by a numerical computation of the eigenmode problem (61) that the behaviours obtained for equilibria \pm 2 extend in a natural manner to higher-order equilibrium states $\pm n$. Thus, equilibria $\pm n$ show $n-1$ unstable eigenmodes (as for the non-linear stability and thermodynamical stability analysis) which can be understood as translations of the density peaks or as slow transfers of matter between neighbouring density peaks. For instance, the equilibrium $n=4$ which corresponds to three density peaks at $x=0, L / 2$ and $L$ exhibits one eigenvalue $v_{1} \simeq 1$ and two positive eigenvalues $v_{2}>v_{3}$ close to zero. For the mode $v_{1}$ the central peak rolls to the left or to the right. For the mode $v_{2}$ which is symmetric with respect to $x=L / 2$ there is a transfer of matter from the central peak towards both left and right peaks (or the opposite since $-q$ is also a growing eigenmode). For the mode $v_{3}$ which is antisymmetric with respect to $x=L / 2$ (for $\delta \eta$ ) there is a transfer of matter from the right peak to the central one and a second transfer with the same amplitude from the central peak to the left one (i.e. a flow from the right to the left or the opposite for $-q$ ). On the other hand, the equilibrium $n=-4$ which corresponds to two density peaks at $x=L / 4$ and $3 L / 4$ exhibits two eigenvalues $v_{1}>v_{2}$ close to unity and one positive eigenvalue $v_{3}$ close to zero. For the mode $v_{1}$ both peaks move with the same velocity towards the same direction while for mode $v_{2}$ they move in opposite directions. The mode $v_{3}$ involves a transfer of matter between both peaks. Note that the largest growing rate corresponds to both peaks moving together in the same direction: the system is not analogous to two particles in the external potential $V$ alone as one needs to take into account the gravitational interaction $\Phi$ which balances $V$ at equilibrium. We would obtain similar results for higher $n$. As noticed above, it would be interesting to follow the evolution of the system by numerical simulations to study the possible merging of these density peaks.

\section{Vlasov equation}

As discussed in Sect. 2 in the mean field limit where the mass of particles goes to zero the $N$-body system (7) is actually described by the Vlasov-Poisson system similar to Eq. (3). As is well-known, the equilibrium states obtained in the hydrodynamical approach of Sect. 4 are also stationary solutions of the Vlasov-Poisson system with the Maxwell-Boltzmann distribution $f(x, v)$ of Eq. (10). Therefore, we recover the equilibrium profiles described in Sect. 3.1.1.

On the other hand, as described in Chavanis (2003) and Tremaine et al. (1986), the Vlasov dynamics conserves any quantity of the form $H[f]=-\int C(f) \mathrm{d} x \mathrm{~d} v$ so that maxima of any functional $H$ where $C(f)$ is convex are non-linearly stable. A particular case of $H[f]$ is the entropy $S[f]$ as given in Eq. (8) hence we can already infer that the equilibrium \pm 1 which is a maximum of $S$ is stable below $T_{c 1}$. However, this criterion cannot decide whether other equilibria $\pm n$ with $n \geq 2$ are stable or not.

Next, in order to study the dynamical linear stability of these equilibrium solutions we linearize the equations of motion. We first introduce the action-angle variables $(J, w)$ which describe the motion of a particle of energy $E$ along its orbit in the equilibrium potential $\phi_{0}(x)$ from position $x_{-}$to $x_{+}$ (see Fridman \& Polyachenko 1984; Polyachenko \& Shukhman 1981):

$J=\frac{2}{2 \pi} \int_{x_{-}}^{x_{+}} \mathrm{d} x \sqrt{2\left(E-\phi_{0}(x)\right)}, \quad w=\frac{\partial S}{\partial J}$,

with:

$S(J, x)=\int_{x_{-}}^{x} \mathrm{~d} x^{\prime} \sqrt{2\left(E-\phi_{0}\left(x^{\prime}\right)\right)}$.

The action-angle variables $(J, w)$ obey Hamilton's equations:

$\dot{w}=\frac{\partial H}{\partial J}=\Omega, \quad \dot{J}=-\frac{\partial H}{\partial w}=0$,

while the Vlasov equation reads:

$\frac{\mathrm{d} f}{\mathrm{~d} t}=\frac{\partial f}{\partial t}+\dot{w} \frac{\partial f}{\partial w}+\dot{J} \frac{\partial f}{\partial J}=0$.

We write the distribution function as $f=f_{0}(J)+f_{1}(J, w)$ where $f_{0}(J)=f_{0}(E)$ is the equilibrium solution and $f_{1}$ the perturbation. Then, the linearized Vlasov equation for $f_{1}$ writes:

$\frac{\partial f_{1}}{\partial t}+\Omega_{0} \frac{\partial f_{1}}{\partial w}=\frac{\partial \phi_{1}}{\partial w} \frac{\mathrm{d} f_{0}}{\mathrm{~d} J}$ 
where $\phi_{1}$ is the perturbed potential. Because of the periodicity of $2 \pi$ with respect to $w$ of the motion of the particles we can write $\phi_{1}$ as:

$\phi_{1}(J, w, t)=\frac{1}{2 \pi} \sum_{l=-\infty}^{\infty} \tilde{\phi}_{1 l}(J) \mathrm{e}^{-\mathrm{i}(\omega t-l w)}$,

where we look for eigenfrequencies $\omega$. Substituting into Eq. (69) yields:

$\tilde{f}_{1}=-\frac{1}{2 \pi} f_{0}^{\prime}(J) \sum_{l} \tilde{\phi}_{1 l} \frac{l}{\omega-l \Omega_{0}} \mathrm{e}^{\mathrm{i} l \omega}$,

with $f_{1}=\tilde{f}_{1} \mathrm{e}^{-\mathrm{i} \omega t}$. Therefore, Poisson's equation reads:

$$
\frac{1}{2 g} \frac{\mathrm{d}^{2} \tilde{\phi}_{1}}{\mathrm{~d} x^{2}}=\tilde{\rho}_{1}=-\frac{1}{2 \pi} \int_{-\infty}^{\infty} \mathrm{d} v f_{0}^{\prime}(J) \sum_{l} \tilde{\phi}_{1 l} \frac{l \mathrm{e}^{\mathrm{i} / \omega}}{\omega-l \Omega_{0}} .
$$

In order to solve the eigenvalue problem (72) it is convenient to project this equation onto a biorthonormalized basis $\left\{\rho_{\alpha}, \phi_{\alpha}\right\}$ such that:

$\frac{\mathrm{d}^{2} \phi_{\alpha}}{\mathrm{d} x^{2}}=2 g \rho_{\alpha}$ and $\int_{0}^{L} \mathrm{~d} x \rho_{\alpha} \phi_{\alpha^{\prime}}=-\delta_{\alpha, \alpha^{\prime}}$,

where $\delta_{\alpha, \alpha^{\prime}}$ is the usual Kronecker symbol (Fridman \& Polyachenko 1984; Kalnajs 1977). For instance, we can choose with $\alpha \geq 1$ :

$\rho_{\alpha}=\frac{\alpha \pi}{\sqrt{g L^{3}}} \cos \left(\alpha \pi \frac{x}{L}\right), \quad \phi_{\alpha}=-2 \frac{\sqrt{g L}}{\alpha \pi} \cos \left(\alpha \pi \frac{x}{L}\right)$,

which also satisfy the boundary conditions $\phi^{\prime}(0)=\phi^{\prime}(L)=0$. Thus, if we decompose $\tilde{\phi}_{1}$ and $\tilde{\rho}_{1}$ as $\tilde{\phi}_{1}=\sum_{\alpha} a_{\alpha} \phi_{\alpha}$ and $\tilde{\rho}_{1}=$ $\sum_{\alpha} a_{\alpha} \rho_{\alpha}$, substituting into Eq. (72) and integrating over $x$ with the weight $\phi_{\alpha}$ yields:

$a_{\alpha}=\int \frac{\mathrm{d} x \mathrm{~d} v}{2 \pi} \phi_{\alpha}(x) f_{0}^{\prime}(J) \sum_{l=-\infty}^{\infty} \sum_{\alpha^{\prime}=1}^{\infty} a_{\alpha^{\prime}}\left(\phi_{\alpha^{\prime}}\right)_{l} \frac{l \mathrm{e}^{\mathrm{i} l \omega}}{\omega-l \Omega_{0}}$.

Using the canonical change of variable $\mathrm{d} x \mathrm{~d} v=\mathrm{d} J \mathrm{~d} w$ and next changing from action $J$ to energy $E$ we obtain for an isothermal equilibrium distribution $f_{0} \propto \mathrm{e}^{-\beta E}$ the eigenvalue problem:

$\operatorname{det}\left(M_{\alpha \alpha^{\prime}}(\omega)-\delta_{\alpha, \alpha^{\prime}}\right)=0$,

with:

$M_{\alpha \alpha^{\prime}}(\omega)=-\frac{\beta}{2 \pi} \int \mathrm{d} E f_{0}(E) \sum_{l} \frac{l}{\omega-l \Omega_{0}}\left(\phi_{\alpha}\right)_{l}\left(\phi_{\alpha^{\prime}}\right)_{l}$.

In terms of the dimensionless variables (14) we obtain for the basis (74):

$M_{\alpha \alpha^{\prime}}=\frac{4 \beta}{\sqrt{\pi} \beta_{c 1}} \int_{\psi_{-}}^{\infty} \mathrm{d} \epsilon \mathrm{e}^{-\epsilon} \tilde{\omega}_{0} \sum_{l=1}^{\infty} \frac{l^{2} \tilde{\omega}_{0}^{2}}{l^{2} \tilde{\omega}_{0}^{2}-\tilde{\omega}^{2}}\left(\hat{\phi}_{\alpha}\right)_{l}\left(\hat{\phi}_{\alpha^{\prime}}\right)_{l}$,

where $\psi_{-}$is the minimum of $\psi(\zeta)$ and with:

$\left(\hat{\phi}_{\alpha}\right)_{l}=\int_{\zeta_{-}}^{\zeta_{+}} \frac{\mathrm{d} \zeta}{\zeta_{L}} \frac{\cos \left(\alpha \pi \zeta / \zeta_{L}\right) \cos \left(l w_{+}\right)}{\alpha \sqrt{\epsilon-\psi}}$.

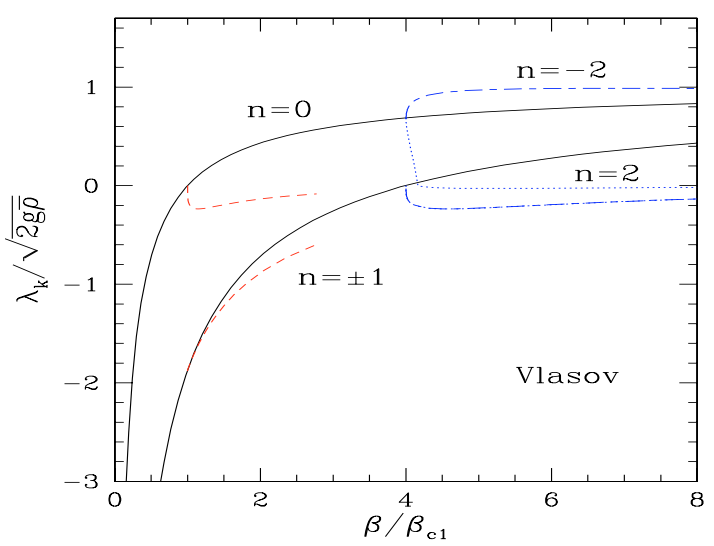

Fig. 12. The stability eigenvalues $\omega_{k} / \sqrt{2 g \bar{\rho}}=i \lambda_{k} / \sqrt{2 g \bar{\rho}}$ as a function of inverse temperature $\beta$ for the equilibria $0, \pm 1$ and \pm 2 for the Vlasov equation of motion. Stability corresponds to $\lambda_{k}<0$. The second eigenvalues $\lambda_{2}$ obtained for equilibria \pm 2 are equal.

Here we introduced the reduced energy $\epsilon=\beta v^{2} / 2+\psi$ of the particle, the frequencies $\tilde{\omega}_{0}$ and $\tilde{\omega}$ defined by:

$\tilde{\omega}=\sqrt{\frac{\beta}{2}} \frac{L}{\pi} \omega=\sqrt{\frac{\beta}{\beta_{c 1}}} \frac{\omega}{\sqrt{4 g \bar{\rho}}}, \quad \tilde{\omega}_{0}=\sqrt{\frac{\beta}{\beta_{c 1}}} \frac{\Omega_{0}}{\sqrt{4 g \bar{\rho}}}$,

and the angular variable $w_{+}$which describes the orbit in the direction of increasing $\zeta$ from $\zeta_{-}$up to $\zeta_{+}$:

$\frac{1}{\tilde{\omega}_{0}}=\int_{\zeta_{-}}^{\zeta_{+}} \frac{\mathrm{d} \zeta}{\zeta_{L}} \frac{1}{\sqrt{\epsilon-\psi}}, \quad w_{+}=\pi \tilde{\omega}_{0} \int_{\zeta_{-}}^{\zeta} \frac{\mathrm{d} \zeta^{\prime}}{\zeta_{L}} \frac{1}{\sqrt{\epsilon-\psi}}$.

For the homogeneous solution we simply have $\psi=0, \tilde{\omega}_{0}=$ $\sqrt{\epsilon}, w_{+}=\pi \zeta / \zeta_{L}$ and $\left(\hat{\phi}_{\alpha}\right)_{l}=\delta_{\alpha, l} /(2 \alpha \sqrt{\epsilon})$. Making the change of variable $x=\sqrt{2 \epsilon}$ we obtain:

$M_{\alpha \alpha^{\prime}}^{0}=\delta_{\alpha, \alpha^{\prime}} \frac{\beta}{\beta_{c 1}} \frac{1}{\alpha^{2} \sqrt{2 \pi}} \int_{-\infty}^{\infty} \mathrm{d} x \mathrm{e}^{-x^{2} / 2} \frac{x}{x-\tilde{\omega} \sqrt{2} / \alpha}$.

Thus, we can see from Eqs. (76), (82), that the condition of marginal stability $\omega=0$ yields again $\beta=\alpha^{2} \beta_{c 1}$ with $\alpha=1,2, \ldots$, which agrees with the critical temperatures (17) obtained in the thermodynamical and hydrodynamical approaches. The matrix $M_{\alpha \alpha^{\prime}}^{0}$ as written above is actually defined for $\operatorname{Im}(\omega)>0$, see Eq. (70), which corresponds to growing modes (instabilities) that vanish for $t \rightarrow-\infty$. For $\operatorname{Im}(\omega)<0$ we need to deform the integration path in Eq. (82) following the usual Landau analysis (e.g. Binney \& Tremaine 1987; Ichimaru 1973). This leads to an additional contribution due to the residue at $x=\tilde{\omega} \sqrt{2} / \alpha$. Thus, for $\operatorname{Im}(\omega)<0$ the matrix $M_{\alpha \alpha^{\prime}}^{0}$ is given by the sum of the expression (82) and of the contribution of the residue which reads:

$\operatorname{Im}(\omega)<0: \quad \operatorname{Res}\left(M_{\alpha \alpha^{\prime}}^{0}\right)=\delta_{\alpha, \alpha^{\prime}} \frac{\beta}{\beta_{c 1}} \frac{2 \sqrt{\pi}}{\alpha^{3}} \mathrm{i} \tilde{\omega} \mathrm{e}^{-\tilde{\omega}^{2} / \alpha^{2}}$.

For the inhomogeneous equilibrium solutions we also need to modify the Eq. (78) in a similar fashion for $\operatorname{Im}(\omega)<0$. However, since we have no analytical expression for the integrand in Eq. (78) this is more difficult. Thus, for numerical purposes we simply add the contribution (83) which is correct close to the critical points. Therefore, the curves in Fig. 12 are 
not correct for $\lambda_{k}<0$ far from the transition $T_{c n}$ but the sign of $\lambda_{k}$ remains exact. Following Kalnajs (1977), in order to improve the convergence with $l$ of the series in Eq. (78) we can write $l^{2} \tilde{\omega}_{0}^{2} /\left(l^{2} \tilde{\omega}_{0}^{2}-\tilde{\omega}^{2}\right)=1+\tilde{\omega}^{2} /\left(l^{2} \tilde{\omega}_{0}^{2}-\tilde{\omega}^{2}\right)$ which leads to

$M_{\alpha \alpha^{\prime}}(\tilde{\omega})=A_{\alpha \alpha^{\prime}}(\tilde{\lambda})+B_{\alpha \alpha^{\prime}}$, where $\tilde{\omega}=\mathrm{i} \tilde{\lambda}$,

with:

$A_{\alpha \alpha^{\prime}}=\frac{-4 \beta}{\sqrt{\pi} \beta_{c 1}} \int_{\psi_{-}}^{\infty} \mathrm{d} \epsilon \mathrm{e}^{-\epsilon} \tilde{\omega}_{0} \sum_{l=0}^{\infty} \frac{\tilde{\lambda}^{2}}{\tilde{\lambda}^{2}+l^{2} \tilde{\omega}_{0}^{2}} \frac{\left(\hat{\phi}_{\alpha}\right)_{l}\left(\hat{\phi}_{\alpha^{\prime}}\right)_{l}}{1+\delta_{l, 0}}$,

and:

$B_{\alpha \alpha^{\prime}}=\frac{2 \beta}{\alpha \alpha^{\prime} \beta_{c 1}} \int \frac{\mathrm{d} \zeta}{\zeta_{L}} \eta(\zeta) \cos \left(\alpha \pi \frac{\zeta}{\zeta_{L}}\right) \cos \left(\alpha^{\prime} \pi \frac{\zeta}{\zeta_{L}}\right)$.

In Eq. (85) the Kronecker factor $\delta_{l, 0}$ reduces the term $l=0$ by a factor 2 as compared with $l \neq 0$. The faster convergence as $1 / l^{2}$ of the expression (85) is convenient for numerical purposes as it reduces the number of terms to be computed. On the other hand, the constant part $B_{\alpha \alpha^{\prime}}$, which does not depend on $\tilde{\lambda}$, is computed separately. Note that $A_{\alpha \alpha^{\prime}}$ does not vanish for $\tilde{\lambda}=0$ because of the term at $l=0$. In practice, in order to solve Eq. (76) we do not need to compute the determinant of the matrix $M_{\alpha \alpha^{\prime}}$. Starting from the transition temperature $T_{c n}$, where the equilibrium solution $\pm n$ which we are interested in separates from the homogeneous equilibrium state, we follow the eigenvalue $v_{k}$ of order $k$ of $M_{\alpha \alpha^{\prime}}$ and the growth rate $\tilde{\lambda}_{k}$ of the associated eigenmode is set by the condition $v_{k}\left(\tilde{\lambda}_{k}\right)=1$ (e.g. Kalnajs 1977). Moreover, contrary to the usual case in plasma physics the eigenfrequencies $\omega$ are imaginary so that $\tilde{\lambda}$ is real. This corresponds to purely growing or decaying modes without oscillatory behaviours. Thus the matrix $M_{\alpha \alpha^{\prime}}(\tilde{\lambda})$ is real and symmetric so that all its eigenvalues $v_{k}$ are real, which simplifies the computation of the roots of $v_{k}\left(\tilde{\lambda}_{k}\right)=1$.

We display in Fig. 12 the stability eigenvalues $\lambda_{k}$ for the equilibria $n=0, \pm 1$ and \pm 2 . We can check that the homogeneous solution $n=0$ shows the same stability properties as those obtained in the thermodynamical and hydrodynamical approaches, as discussed below Eq. (82). On the other hand, from Eq. (82) we can see that at low temperatures we have for the highest eigenvalue $\tilde{\lambda}_{1}$ the asymptotic behaviour $\tilde{\lambda}_{1} \simeq \sqrt{\beta / 2 \beta_{c 1}}$ which yields $\lambda_{1} \rightarrow \sqrt{2 g \bar{\rho}}$. For the equilibrium $n= \pm 1$ we only plot the curves up to $3 \beta_{c 1}$ for clarity and because our formula (83) for the residue contribution only holds close to $T_{c 1}$. However, we checked numerically that $\lambda_{k}$ remains negative at low $T$ (the approximation (83) only enters for $\lambda_{k}<0$ so that the sign of the eigenvalues $\lambda_{k}$ does not depend on it). Therefore, as for the thermodynamical and hydrodynamical approaches the states \pm 1 are stable below $T_{c 1}$.

In a similar fashion, the equilibrium $n=-2$ (which corresponds to a density peak at $x=L / 2$ ) shows one unstable mode as in Fig. 9 with $\lambda_{1} / \sqrt{2 g \bar{\rho}} \rightarrow 1$ at low temperature. However, Fig. 13 shows that the corresponding eigenmode is significantly different from the one obtained in the hydrodynamical framework (compare with Fig. 10). Indeed, the growing mode $\delta \eta$ no longer tends to the derivative $\eta^{\prime}$ associated with a pure translation of the central density peak. Although close to the peak at $x=L / 2$ it can be interpreted as a translation the perturbation

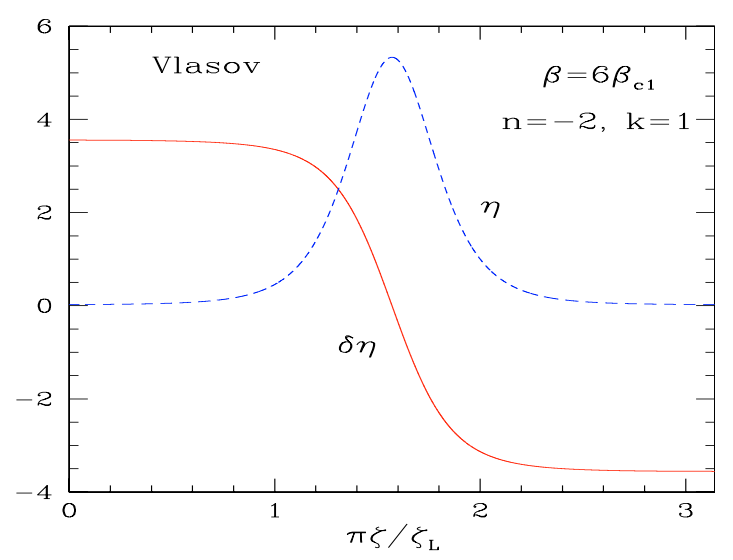

Fig. 13. The eigenmode $k=1$ for the equilibrium solution $n=-2$ at temperature $\beta=6 \beta_{c 1}$. We plot both the equilibrium profile $\eta$ (dashed line) and its unstable mode $\delta \eta$ (solid line) with eigenvalue $\lambda_{1}>0$.

extends as far as the boundaries $x=0, L$, so that it also involves a deformation of the density profile. Therefore the dynamics of the relaxation towards the stable equilibrium \pm 1 proceeds in a manner which is different from the behaviour obtained within the hydrodynamical model. In particular, the deformation implied by this instability suggests that a solitonic behaviour cannot arise as some mass leaks from the central peak down to the boundary $x=0$ while the peak moves towards $x=0$. This difference with respect to the hydrodynamical case may be understood as follows. In the collisional fluid model the evolution proceeds in a well-ordered manner through local processes, as each fluid element at location $x$ reacts to slow changes in the local pressure and density. By contrast, in the collisionless case described by the Vlasov equation particles probe far away regions as their orbit extends to both sides of the density peak. Thus, as the central peak moves towards $x=0$ as in Fig. 13 the left/right symmetry is broken and the potential $\phi$ becomes deeper to the left of the peak. Then, if we consider a subsample of particles which relax to the new potential as $\rho_{\text {sub }} \propto \mathrm{e}^{-\phi}$, as they can move from one side of the density peak to the other, they will populate the left side of the peak more than the right side. This yields $\delta \eta(0)>0>\delta \eta(L)$ as in Fig. 10 (of course the collisionless Vlasov dynamics conserves entropy but such a relaxation may be applied to the coarse-grained distribution which mixes the two subsamples).

Finally, the equilibrium $n=2$ also shows at first an unstable mode $\lambda_{1}$ which quickly goes towards zero as in Fig. 9 but contrary to the hydrodynamical case the eigenvalue $\lambda_{1}$ becomes negative which means that at low $T$ this state turns stable. The density profile of this eigenmode displayed in Fig. 14 at $6 \beta_{c 1}$ shows that it is again significantly different from its hydrodynamical counterpart. Indeed, as for equilibrium -2 it exhibits a broader extension than within the hydrodynamical framework which is related to the collisionless nature of the system which entails changes on a more global scale. This implies that $\eta+\delta \eta$ does not correspond to a quasi-static evolution where both density peaks would follow equilibrium profiles with different slowly varying masses. Thus, it appears that the decrease of entropy $S[f]$, understood as an $\mathrm{H}$-function as in Chavanis (2003), due to the distortion of the profiles close to 


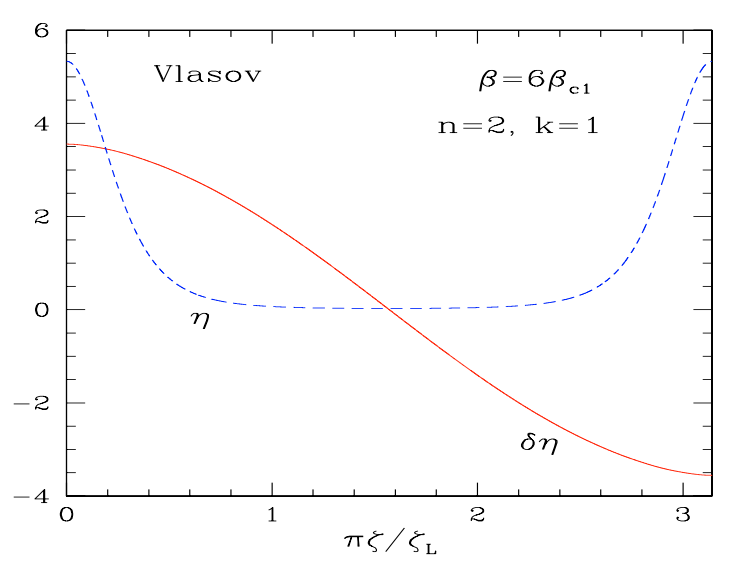

Fig. 14. The eigenmode $k=1$ for the equilibrium solution $n=2$ at temperature $\beta=6 \beta_{c 1}$. We plot both the equilibrium profile $\eta$ (dashed line) and its least stable mode $\delta \eta$ (solid line) with eigenvalue $\lambda_{1} \simeq 0$.

the boundaries $x=0, L$, farther from isolated equilibrium states dominates the increase of entropy which would be associated with the transformation of the system from two separate density peaks towards only one density peak (state \pm 1 which exhibits a higher $S[f]$, as seen from Fig. 2). Thus, thermodynamical considerations or non-linear analysis based on $\mathrm{H}$-functions are not sufficient to obtain the dynamical stability properties of the system. One needs to take into account the constraints brought by the Vlasov dynamics: one cannot select any arbitrary deformation path from equilibrium 2 towards equilibrium \pm 1 . As discussed in Lynden-Bell (1967), the infinite number of conserved quantities associated with the collisionless dynamics (the total mass of phase-space element greater than any $f$ is conserved) can actually be taken into account by using a new "collisionless entropy" ln $W$ which differs from the usual Boltzmann entropy. On the other hand, the comparison with Sect. 4 shows that the collisional and collisionless dynamics are significantly different, as it appears that the hydrodynamical dynamics allows the system to flow from state $n=2$ to state $n= \pm 1$ (which implies that equilibrium $n=2$ is unstable) whereas at low $T$ the Vlasov dynamics prevents the system from going to $n= \pm 1$ from $n=2$ through linear instabilities (but clearly a non-linear perturbation can lead to relaxation towards $n= \pm 1$ ).

\section{Conclusion}

We have studied in this article the thermodynamical properties of a 1-D gravitational system built from the problem of largescale structure formation in cosmology. We have considered time-scales much smaller than the Hubble time so that the expansion of the universe can be neglected and the system can be restricted to a finite size $L$. Then, the cosmological background merely yields an effective external potential $V$ in addition to the gravitational self-interaction $\Phi$ so that an homogeneous equilibrium (corresponding to the Hubble flow) exists at all temperature $T$. We have found that all three micro-canonical, canonical and grand-canonical ensembles give the same results. There exists a series of critical temperatures $T_{c n}\left(T_{c 1}>T_{c 2}>\ldots\right)$ below which two new equilibria $\pm n$ appear, reflecting the usual Jeans instability mechanism. Above $T_{c 1}$ the homogeneous state is thermodynamically stable whereas below $T_{c 1}$ it is unstable and only equilibria \pm 1 (corresponding to a density peak at the boundary $x=0$ or $L$ ) are stable while other states $\pm n$ with $n \geq 2$ are unstable. The system shows a second-order phase transition at $T_{c 1}$ where the specific heat is discontinuous.

Next, we have studied the hydrodynamical properties of these equilibria. We have found that both the non-linear stability and the dynamical linear stability coincide with the results of the thermodynamical analysis. The evolution of the system can be understood in a simple manner from the translation and diffusion of the various density peaks associated with states $\pm n$ which would relax towards the stable equilibria \pm 1 . Finally, we have studied the collisionless dynamics governed by the Vlasov equation. The equilibria \pm 1 are again both linearly and nonlinearly stable while the homogeneous state is unstable below $T_{c 1}$. We found that the equilibrium $n=-2$ (one density peak at $x=L / 2$ ) is again unstable but the equilibrium $n=2$ (two density peaks located at both boundaries) shows a new behaviour. Although it is unstable just below $T_{c 2}$ as in the thermodynamical and hydrodynamical approaches it now turns stable at low temperatures. This departure from the results of the thermodynamical and hydrodynamical analysis is due to the dynamical constraints associated with the equations of motion of a collisionless system. On the other hand, other equilibria $\pm n$ with $n \geq 3$ are again unstable. Therefore, depending on the initial conditions the system would relax towards equilibria \pm 1 or 2 (i.e. a density peak at either one or both boundaries).

Thus, the behaviour of the 1-D gravitational system (OSC) studied in this article is quite similar to the HMF model, described for instance in Chavanis et al. (2005). We obtain the same second-order phase transition from a high-temperature homogeneous equilibrium to a clustered equilibrium \pm 1 at low temperature. The stability properties of these two configurations are also similar, for all three thermodynamical, hydrodynamical and collisionless analysis. However, in the case of the OSC system the scale-free nature of the gravitational interaction leads to an additional series of equilibria $\pm n(n \geq 2)$. These new states are unstable, except for the equilibrium $n=2$ which turns linearly stable for a collisionless dynamics at low $T$, but remains unstable for both the thermodynamical and hydrodynamical approaches. This provides an interesting case where the collisionless dynamics exhibits a specific behaviour and leads to a relaxation which clearly depends on initial conditions. On the other hand, it would be interesting to study the non-linear evolution of the system and the possible merging of several density peaks in both the hydrodynamical and collisionless cases. This might be studied through $N$-body simulations as in Hohl \& Feix (1967) or through a direct computation of the Vlasov dynamics as in Alard \& Colombi (2005). Finally, one should include the expansion of the universe to study the non-equilibrium dynamics associated with cosmological hierarchical scenarios. This is left for future studies but we can expect this 1-D gravitational system to provide a useful tool to investigate such processes.

\section{References}

Alard, C., \& Colombi, S. 2005, MNRAS, 359, 123

Aurell, E., \& Fanelli, D. 2001, [cond-mat/0106444] 
Aurell, E., Fanelli, D., \& Muratore-Ginanneschi, P. 2001, Physica D, 148, 272

Bernardeau, F. 1992, ApJ, 392, 1

Binney, J., \& Tremaine, S. 1987, Galactic dynamics (Princeton University Press)

Bond, J. R., Cole, S., Efstathiou, G., \& Kaiser, N. 1991, ApJ, 379, 440

Camm, G. L. 1950, MNRAS, 110, 305

Chandrasekhar, S. 1942, An introduction to the theory of stellar structure, Dover

Chavanis, P. H. 2002, A\&A, 381, 340

Chavanis, P. H. 2003, A\&A, 401, 15

Chavanis, P. H. 2006, Physica A, 361, 81

Chavanis, P. H., Vatteville, J., \& Bouchet, F. 2005, Eur. Phys. J. B, 46, 61

de Vega, H. J., \& Siebert, J. A. 2005a, Nucl. Phys. B, 707, 529

de Vega, H. J., \& Siebert, J. A. 2005b, Nucl. Phys. B, 726, 464

Fanelli, D., \& Aurell, E. 2002, A\&A, 395, 399

Fridman, A. M., \& Polyachenko, V. L. 1984, Physics of gravitating systems (Springer-Verlag)

Fry, J. N. 1984, ApJ, 279, 499

Goroff, M. H., Grinstein, B., Rey, S.-J., \& Wise, M. B. 1986, ApJ, 311, 6

Gurbatov, S. N., Saichev, A. I., \& Shandarin, S. F. 1989, MNRAS, 236, 385
Hohl, F., \& Feix, M. R. 1967, ApJ, 147, 1164

Ichimaru, S. 1973, Basic principles of plasma physics, Frontiers in physics (the Benjamin/Cummings publish. comp.)

Kalnajs, A. J. 1977, ApJ, 212, 637

Koyama, H., \& Konishi, T. 2001, Phys. Lett. A, 279, 226

Luwel, M., \& Severne, G. 1985, A\&A, 152, 305

Lynden-Bell, D. 1967, MNRAS, 136, 101

Munshi, D., Bernardeau, F., Melott, A. L., \& Schaeffer, R. 1999, MNRAS, 303, 433

Padmanabhan, T. 1990, Phys. Rep., 188, 285

Peebles, P. J. E. 1980, The large scale structure of the universe (Princeton University Press)

Peebles, P. J. E. 1982, ApJ, 263, L1

Polyachenko, V. L., \& Shukhman, I. G. 1981, Sov. Astron., 25, 533

Press, W. H., \& Schechter, P. 1974, ApJ, 187, 425

Rouet, J. L., \& Feix, M. R. 1999, Phys. Rev. E, 59, 73

Semelin, B., de Vega, H. J., Sanchez, N., \& Combes, F. 1999, Phys. Rev. D, 59, 125021

Shandarin, S. F., \& Zeldovich, Y. B. 1989, Rev. Mod. Phys., 61, 185

Tremaine, S., Henon, M., \& Lynden-Bell, D. 1986, MNRAS, 219, 285

Valageas, P. 2001, A\&A, 379, 8

Valageas, P. 2002, A\&A, 382, 450

Valageas, P. 2004, A\&A, 421, 23

Youngkins, V. P., \& Miller, B. N. 2000, Phys. Rev. E, 62, 4583 